\title{
Common Fixed Point Theorems for Weakly Contractions in Rectangular b-Metric Spaces with Supportive Applications
}

\author{
Hongyan Guan $(\mathbb{D}$, Jianju Li $\mathbb{D}$, and Yan Hao $\mathbb{1}$ \\ School of Mathematics and Systems Science, Shenyang Normal University, Shenyang 110034, China \\ Correspondence should be addressed to Yan Hao; haoyan8012@163.com
}

Received 9 November 2021; Revised 17 December 2021; Accepted 20 December 2021; Published 13 January 2022

Academic Editor: Hüseyin Işık

Copyright (c) 2022 Hongyan Guan et al. This is an open access article distributed under the Creative Commons Attribution License, which permits unrestricted use, distribution, and reproduction in any medium, provided the original work is properly cited.

\begin{abstract}
In this manuscript, two new classes of generalized weakly contractions are introduced and common fixed point results concerning the new contractions are proved in the context of rectangular $b$-metric spaces. Also, some examples are included to present the validity of our theorems. As an application, we provide the existence and uniqueness of solution of an integral equation.
\end{abstract}

\section{Introduction}

In the field of nonlinear analysis, Banach fixed point theorem, which is introduced by Banach [1], is a powerful and classical means to deal with problems on fixed points in metric spaces. It is widely used in many disciplines of mathematics and has been promoted in many aspects. One important extension is to extend the concept of metric spaces. $b$ -metric spaces and rectangular metric spaces are regarded as two well-known generalizations of metric spaces.

As a extension of a metric space, $b$-metric space was firstly introduced by Czerwik [2], by modifying the third condition of metric function. In that paper, the author provided fixed point results for contraction conditions in this type space. Afterwards, some authors have obtained many excellent results concerning fixed point theory of a lot of new types of contractive mappings on $b$-metric spaces. Generalizing the results of Berinde [3], Zada et al. [4] obtained fixed point results for mappings with rational type and Pacurar [5] got fixed point theorems of $\varphi$-contractions. In [6], common fixed point results for weak $\varphi$-contraction mappings were proved in this type spaces by Aydi et al. In 2019, problems about periodic common fixed point were studied by Hussain et al. [7]. Recently, in [8], Gopal et al. explored the latest researches and developments on theory of fixed point in the framework of $b$-metric spaces. Younis et al. [9] introduced new fixed point results for the underlying mappings in the framework of dislocated $b$-metric spaces. In [10], in $b$-metric-like spaces, the authors extended the concept of Kannan mappings in view of $F$-contraction. Lately, Younis et al. [11] presented the notion of graphical extended $b$-metric spaces and discussed the framework of an open ball in this new type space.

In 2000 , by changing triangular inequality to quadrilateral inequality, more general inequality, Branciari [12] introduced the concept of rectangular metric spaces. Also, the author extended the Banach contraction mapping principle for this new context. Subsequently, a lot of fixed point theorems of various contractive conditions in rectangular metric spaces were obtained. Lakzian et al. [13] established fixed point theorems dealing with $(\psi, \phi)$ -weakly contraction conditions in this type space, which was ulteriorly extended by Erhan et al. in [14]. Bari and Vetro [15] got common fixed point results on given functions with $(\psi, \phi)$-weakly contractive conditions. In [16], George and Rajagopalan studied problems of common fixed points of $(\psi, \phi)$-contractive mappings. Lately, in complete rectangular metric spaces, Wang and Pei-Sheng [17] gave generalised $\theta$-contraction mappings which can be regarded as generalized Suzuki-Berinde type $\theta$-contraction mappings and provided conditions which ensured this type mapping possesses a unique fixed point. By the 
help of $C$-functions, in [18], some fixed point results were established by Budhia et al. In graphical rectangular $b$ -metric spaces, some errors from literature [19] were rectified by Younis et al. in [20].

Inspired by results of Czerwik [2] and Branciari [12], George et al. [21] extended $b$-metric space and rectangular metric space by introduced rectangular $b$-metric space. In that paper, the authors presented an analogue of Banach fixed point theorem and fixed point theorem of Kannan. After that, many researchers had solved problems of fixed point of new type of contractive mappings on this type space. Kadelburg and Radenovic [22] and Mitrovic [23] presented common fixed point theorems in this type space. In the setting of rectangular $b$-metric spaces, a Boyd-Wong type theorem was studied by Ding et al. in [24]. Sukprasert et al. [25] presented the concept of weak altering distance function and discussed fixed point result of a new generalized contractive mapping. Roshan et al. [26] gave some fixed point theorems concerning almost generalized weakly contractive mappings and rational type contractions. In [27], Mitrovic obtained an analogue of Banach contractive mapping principle and solved an open problem arose in [21].
Recently, Sunarsini et al. [28] introduced a new extension of metric space named as complex valued rectangular $b$ - metric space and gave an example of Banach contractive mapping principle at linear equation system. In [29], common coupled fixed point theorems concerning generalised $T$-contraction conditions were studied by George and Reshma. Lately, in ordered partial rectangular $b$-metric spaces, Asim et al. [30] established some orderedtheoretic fixed point results of Geraghty-weak contractive mappings.

In 1997, by using the notion of weak contractive mappings, Alber et al. [31] extended Banach contraction mapping principle in Hilbert spaces. In [32], weak contraction principle was generalized to metric spaces by Rhoades. After that, many authors had generalised the weak contraction principle. For example, in [33], the authors obtained the fixed point results involving $\alpha-\psi$ contraction conditions and applied them to solve quadratic integral equations. In [34], Jamal et al. used $(\psi, \phi)$-weak contraction to extend coincidence point theorems obtained in partially ordered $b$ -metric spaces.

Set

$$
\begin{gathered}
\Psi=\{\psi:[0,+\infty) \longrightarrow[0,+\infty) \text { is a continuous and increasing function }\} \\
\Phi=\{\phi:[0,+\infty) \longrightarrow[0,+\infty) \text { is a lower semi continuous and nondecreasing function and } \phi(t)=0 \text { if and only if } t=0\} .
\end{gathered}
$$

Hao and Guan [35] proved common fixed point result dealing with a new class of generalized weakly contraction conditions in complete $b$-metric spaces as follows.

Theorem 1 (see [35]). Let $(\mathscr{E}, \rho)$ be a complete $b$-metric space with coefficient $s \geq 1$. Let $O, R: \mathscr{E} \longrightarrow \mathscr{E}$ be self-mappings such that $R$ is injective and $O(\mathscr{E}) \subset R(\mathscr{E})$ where $R(\mathscr{E})$ is closed. Assume that $p \geq 2$ is a fixed number and $\varphi: \mathscr{E}$ $\longrightarrow[0,+\infty)$ is lower semicontinuous. If there exist $\psi \in \Psi$ and $\phi \in \Phi$ satisfying

$$
\begin{aligned}
& \psi\left(s^{p}[\rho(O \xi, O \eta)+\varphi(O \xi)+\varphi(O \eta)]\right) \\
& \quad \leq \psi(I(\xi, \eta, \rho, O, R, \varphi))-\phi(J(\xi, \eta, \rho, O, R, \varphi)),
\end{aligned}
$$

where

$$
\begin{aligned}
I(\xi, \eta, \rho, O, R, \varphi)= & \max \left\{\rho(R \xi, R \eta)+\varphi(R \xi)+\varphi(R \eta), \frac{1}{2}\{\rho(O \xi, R \xi)+\varphi(O \xi)\right. \\
+ & \varphi(R \xi)+\rho(O \eta, R \eta)+\varphi(O \eta)+\varphi(R \eta)\}, \frac{1}{2 s}\{\rho(O \xi, R \eta) \\
+ & \varphi(O \xi)+\varphi(R \eta)+\rho(O \eta, R \xi)+\varphi(O \eta)+\varphi(R \xi)\}\}, \\
J(\xi, \eta, \rho, O, R, \varphi)= & \max \{\rho(R \xi, R \eta)+\varphi(R \xi)+\varphi(R \eta), \rho(O \eta, R \eta) \\
& +\varphi(O \eta)+\varphi(R \eta)\},
\end{aligned}
$$

then $O$ and $R$ possess a unique coincidence point in $\mathscr{E}$. Fur- ther, if $O$ and $R$ are weakly compatible, then $O$ and $R$ have a unique common fixed point.

Continuing in the same direction, our aim is to give two new classes of generalized weakly contractions and establish some common fixed point theorems dealing with the new contractions in the setting of rectangular $b$-metric spaces. Moreover, we present some examples that elaborate the validity of our theorems. Also, as an application, we prove the existence of solution of an integral equation.

\section{Preliminaries}

First, we recall some definitions and lemmas as follows:

Definition 2 (see [2]). Let $\mathscr{M}$ be a nonempty set and $s \geq 1$ be a constant. A function $\sigma: \mathscr{M} \times \mathscr{M} \longrightarrow[0,+\infty)$ is said to be a $b$-metric iff

(i) $\sigma(\xi, \eta)=0$ iff $\xi=\eta$ for $\xi, \eta \in \mathscr{M}$

(ii) $\sigma(\xi, \eta)=\sigma(\eta, \xi)$ for $\xi, \eta \in \mathscr{M}$

(iii) there exists a real number $s \geq 1$ satisfying $\sigma(\xi, \eta) \leq$ $s(\sigma(\xi, \vartheta)+\sigma(\eta, \vartheta))$ for $\xi, \eta, \vartheta \in \mathscr{M}$

Usually, we call $(\mathscr{M}, \sigma)$ a $b$-metric space with coefficient $s \geq 1$. 
Definition 3 (see [12]). Let $\mathscr{M}$ be a nonempty set. A function $\rho: \mathscr{M} \times \mathscr{M} \longrightarrow[0,+\infty)$ is said to be a rectangular metric iff

(i) $\rho(\xi, \eta)=0$ iff $\xi=\eta$ for $\xi, \eta \in \mathscr{M}$

(ii) $\rho(\xi, \eta)=\rho(\eta, \xi)$ for $\xi, \eta \in \mathscr{M}$

(iii) $\rho(\xi, \eta) \leq \rho(\xi, \kappa)+\rho(\kappa, v)+\rho(\nu, \eta)$ for $\xi, \eta \in \mathscr{M}$ and all different points $\kappa, v \in \mathscr{M}-\{\xi, \eta\}$

In general, we call $(\mathscr{M}, \rho)$ a rectangular metric space.

Definition 4 (see [21]). Let $\mathscr{M}$ be a nonempty set and $s \geq 1$ be a constant. A function $\mathrm{Q}: \mathscr{M} \times \mathscr{M} \longrightarrow[0,+\infty)$ is said to be a rectangular $b$-metric iff

(i) $\mathrm{\varrho}(\xi, \eta)=0$ iff $\xi=\eta$ for $\xi, \eta \in \mathscr{M}$

(ii) $\mathrm{Q}(\xi, \eta)=\mathrm{Q}(\eta, \xi)$ for $\xi, \eta \in \mathscr{M}$

(iii) there exists a real number $s \geq 1$ satisfying $\mathrm{Q}(\xi, \eta) \leq$ $s(\mathrm{Q}(\xi, \kappa)+\mathrm{Q}(\kappa, v)+\mathrm{Q}(v, \eta))$ for $\xi, \eta \in \mathscr{M}$ and all different points $\kappa, v \in \mathscr{M}-\{\xi, \eta\}$

As usual, we call $(\mathscr{M}, \mathrm{\varrho})$ a rectangular $b$-metric space with coefficient $s \geq 1$.

Remark 5. It is obvious that a rectangular metric function becomes a metric function when $\kappa=v$ and a rectangular $b$ -metric function becomes a rectangular metric function when $s=1$, whereas the converse of this statement may not be true (see [21], Examples 1.4 and 1.5).

Example 1. Let $\mathscr{M}=A \cup B$, where $A=\{1 / 2,1 / 3,1 / 4,1 / 5\}, B$ $=[1,2]$. Define $\mathrm{\varrho}: \mathscr{M} \times \mathscr{M} \longrightarrow[0,+\infty)$ with $\mathrm{\varrho}(\xi, \eta)=\mathrm{\varrho}(\eta, \xi$ ) for $x, y \in X$ and

$$
\left\{\begin{array}{l}
\varrho\left(\frac{1}{2}, \frac{1}{3}\right)=\varrho\left(\frac{1}{3}, \frac{1}{4}\right)=\varrho\left(\frac{1}{4}, \frac{1}{5}\right)=0.05, \\
\varrho\left(\frac{1}{2}, \frac{1}{4}\right)=\varrho\left(\frac{1}{3}, \frac{1}{5}\right)=0.08, \\
\varrho\left(\frac{1}{2}, \frac{1}{5}\right)=0.6, \\
\varrho(\xi, \eta)=|\xi-\eta|, \text { otherwise. }
\end{array}\right.
$$

By calculation, we get $(\mathscr{M}, \mathrm{\varrho})$ is a rectangular $b$-metric space as $s=4$, whereas we obtain the following results:

(1) $(\mathscr{M}, \varrho)$ is not a metric space, as

$$
\mathrm{\varrho}\left(\frac{1}{2}, \frac{1}{5}\right)=0.6>0.13=\mathrm{\varrho}\left(\frac{1}{2}, \frac{1}{3}\right)+\mathrm{\varrho}\left(\frac{1}{3}, \frac{1}{5}\right)
$$

(2) $(\mathscr{M}, \varrho)$ is not a rectangular metric space, as

$$
\mathrm{Q}\left(\frac{1}{2}, \frac{1}{5}\right)=0.6>0.15=\varrho\left(\frac{1}{2}, \frac{1}{3}\right)+\mathrm{Q}\left(\frac{1}{3}, \frac{1}{4}\right)+\varrho\left(\frac{1}{4}, \frac{1}{5}\right)
$$

(3) $(\mathscr{M}, \varrho)$ is not a $b$-metric space with $s=4$, as

$$
\mathrm{\varrho}\left(\frac{1}{2}, \frac{1}{5}\right)=0.6>0.52=4 \cdot\left(\mathrm{\varrho}\left(\frac{1}{2}, \frac{1}{3}\right)+\mathrm{\varrho}\left(\frac{1}{3}, \frac{1}{5}\right)\right)
$$

Example 2. Assume $\left(\mathscr{M}, \mathrm{Q}^{*}\right)$ is a metric space. For $p \geq 2$, define $\mathrm{\varrho}(\xi, \eta)=\left(\mathrm{\varrho}^{*}(\xi, \eta)\right)^{p}$. Then, $(\mathscr{M}, \mathrm{\varrho})$ is a rectangular $b$ -metric space with parameter $s=3^{p-1}$.

Proof. One can verify easily the conditions (i) and (ii) hold by definition of $\mathrm{Q}(\xi, \eta)$. In order to check (iii), we can infer from the following inequality:

$(m+n+l)^{p} \leq 3^{p-1}\left(m^{p}+n^{p}+l^{p}\right)$, for any $m, n, l \geq 0$ and $p \geq 2$.

Then, for $\xi, \eta \in \mathscr{M}$ and all different points $\tau, v \in \mathscr{M}-\{\xi$ $, \eta\}$, we have

$$
\begin{aligned}
\mathrm{\varrho}(\xi, \eta) & =\left(\mathrm{\varrho}^{*}(\xi, \eta)\right)^{p} \leq\left(\mathrm{\varrho}^{*}(\xi, \tau)+\mathrm{Q}^{*}(\tau, v)+\mathrm{Q}^{*}(v, \eta)\right)^{p} \\
& \leq 3^{p-1}(\mathrm{\varrho}(\xi, \tau)+\mathrm{\varrho}(\tau, v)+\mathrm{\varrho}(v, \eta)) .
\end{aligned}
$$

That is, $(\mathscr{M}, \mathrm{Q})$ is a rectangular $b$-metric space when $s$ $=3^{p-1} \cdot \square$

Definition 6 (see [21]). Let $(\mathscr{M}, \mathrm{\varrho})$ be a rectangular $b$-metric space with coefficient $s \geq 1$. A sequence $\left\{\xi_{n}\right\}$ in $\mathscr{M}$ is called:

(i) convergent sequence iff there is $\xi \in \mathscr{M}$ such that $\mathrm{Q}($ $\left.\xi_{n}, \xi\right) \longrightarrow 0$ as $n \longrightarrow+\infty$

(ii) a Cauchy sequence iff $\mathrm{Q}\left(\xi_{n}, \xi_{m}\right) \longrightarrow 0$ when $n, m$ $\longrightarrow+\infty$

Furthermore, a rectangular $b$-metric space is called completeness iff every Cauchy sequence is convergent.

Remark 9. In rectangular $b$-metric spaces, one can show that the limit of a sequence may not unique and every convergent sequence in a rectangular $b$-metric space may not be a Cauchy sequence(see [21], Example 1.7). 
Definition 8 (see [36]). Let $O$ and $R$ be two self-maps defined on a nonempty set $\mathscr{M}$. If $v=O \xi=R \xi$, for some $\xi \in \mathscr{M}$, then $\nu$ is called the point of coincidence of $O$ and $R$, where $\xi$ is said to be the coincidence point of $O$ and $R$. Let $C(O, R)$ represent the collection of all coincidence points of $O$ and $R$.

Definition 9 (see [36]). Let $O$ and $\mathrm{R}$ be two self-maps defined on a nonempty set $\mathscr{M}$. Then, $O$ and $R$ are called weakly compatible mappings when they commute at each coincidence point, i.e., $O \xi=R \xi \Rightarrow O R \xi=R O \xi$ for each $\xi \in C(O, R)$.

Lemma 10 (see [26]). Let $(\mathscr{M}, \mathrm{Q})$ be a rectangular $b$-metric space with parameter $s \geq 1$. Assume that $\left\{\xi_{n}\right\}$ and $\left\{\eta_{n}\right\}$ are convergent to $\xi$ and $\eta$, respectively. Then, one can get

$$
\frac{1}{s} \mathrm{Q}(\xi, \eta) \leq \liminf _{n \longrightarrow+\infty} \mathrm{\varrho}\left(\xi_{n}, \eta_{n}\right) \leq \limsup _{n \longrightarrow+\infty} \mathrm{Q}\left(\xi_{n}, \eta_{n}\right) \leq s \mathrm{\varrho}(\xi, \eta) .
$$

Moreover, if $\xi=\eta$, then we have $\lim _{n \longrightarrow+\infty} \mathrm{\varrho}\left(\xi_{n}, \eta_{n}\right)=0$. Further, for $\zeta \in \mathscr{M}$, we deduce

$$
\frac{1}{s} \mathrm{Q}(\xi, \zeta) \leq \liminf _{n \longrightarrow+\infty} \mathrm{Q}\left(\xi_{n}, \zeta\right) \leq \limsup _{n \longrightarrow+\infty} \mathrm{Q}\left(\xi_{n}, \zeta\right) \leq s \mathrm{\varrho}(\xi, \zeta)
$$

\section{Main Results}

In this section, a few of new common fixed point results on generalized weakly contractive conditions in a complete rectangular $b$-metric space will be presented. Moreover, two examples will be provided to prove the validity of our theorems.

Suppose $(\mathscr{M}, \varrho)$ is a rectangular $b$-metric space. A mapping $O: \mathscr{M} \longrightarrow[0,+\infty)$ is named as a lower semicontinuous mapping if, for $\xi \in \mathscr{M}$ and $\left\{\xi_{n}\right\}$ is convergent to $\xi$, one get

$$
O(\xi) \leq \liminf _{n \longrightarrow+\infty} O\left(\xi_{n}\right)
$$

Let $\Omega$ represent the set of all functions $\beta: \mathbb{R}_{0}{ }^{+} \longrightarrow[0,1$ $/ s)$. We shall consider the contractive conditions defined by the family $\Theta$ :

$$
\begin{aligned}
\Theta & =\{\theta:[0,+\infty) \longrightarrow[0,+\infty) \text { is a continuous and increasing function, for all } \kappa \\
& >0, \theta(\kappa)<\kappa \text { and } \theta(\kappa)=0 \text { iff } \kappa=0\} .
\end{aligned}
$$

Lemma 11 (see [37]). Let $\theta:[0,+\infty) \longrightarrow[0,+\infty)$ be a nondecreasing and upper semicontinuous mapping. Then, $\theta(x)$ $<x$ for any $x>0$ iff $\theta^{n}(x) \longrightarrow 0$ as $n \longrightarrow \infty$.

Definition 12. Let $(\mathscr{M}, \rho)$ be a rectangular $b$-metric space with coefficient $s \geq 1$. Let $\alpha: \mathscr{M} \times \mathscr{M} \longrightarrow[0,+\infty)$ and $O, R$ $: \mathscr{M} \longrightarrow \mathscr{M}$ be given functions and $p \geq 2$ be a real number. A function $O: \mathscr{M} \longrightarrow \mathscr{M}$ is called $R-\alpha_{s^{p}}$-admissible function if, for all $\xi, \eta \in \mathscr{M}, \alpha(R \xi, R \eta) \geq s^{p}$ implies $\alpha(O \xi, O \eta) \geq s^{p}$.

Definition 13. Let $(\mathscr{M}, \mathrm{Q})$ be a rectangular $b$-metric space with coefficient $s \geq 1$. Let $\alpha: \mathscr{M} \times \mathscr{M} \longrightarrow[0,+\infty)$ and $O, R$
$: \mathscr{M} \longrightarrow \mathscr{M}$ be three given mappings. Suppose that $p \geq 2$ is a real number and $\varphi: \mathscr{M} \longrightarrow[0,+\infty)$ is a lower semicontinuous function. A mapping $O$ is called a generalized $\left(R-\alpha_{s^{p}}\right.$ $, \theta, \varphi)$ contractive mapping, if there exist $\theta \in \Theta, \beta \in \Omega$ and $L \geq 0,1 / s+L<1$ satisfying

$$
\begin{aligned}
& \theta(\alpha(R \xi, R \eta)[\varrho(O \xi, O \eta)+\varphi(O \xi)+\varphi(O \eta)]) \\
& \quad \leq \beta(\theta(h(\xi, \eta, \varrho, O, R, \varphi))) \theta(h(\xi, \eta, \varrho, O, R, \varphi)) \\
& \quad+L \theta(q(\xi, \eta, \varrho, O, R, \varphi))
\end{aligned}
$$

for all $\xi, \eta \in \mathscr{M}$ with $\alpha(R \xi, R \eta) \geq s^{p}$ and $\mathrm{Q}(O \xi, O \eta)+\varphi(O \xi)$ $+\varphi(O \eta) \neq 0$, where

$$
\begin{aligned}
h(\xi, \eta, \mathrm{e}, O, R, \varphi)= & \max \left\{\mathrm{e}(O \xi, O \eta)+\varphi(O \xi)+\varphi(O \eta), \frac{\mathrm{\varrho}(O \eta, O \eta)+\varphi(O \eta)+\varphi(R \eta)}{1+\mathrm{e}(O \xi, R \xi)+\varphi(O \xi)+\varphi(R \xi)}\right. \\
& \cdot\{\mathrm{e}(R \xi, R \eta)+\varphi(R \xi)+\varphi(R \eta)\}, \frac{1}{2}\{\mathrm{e}(O \xi, O \eta)+\varphi(O \xi)+\varphi(O \eta) \\
& +\mathrm{\varrho}(R \xi, R \eta)+\varphi(R \xi)+\varphi(R \eta)\}\},
\end{aligned}
$$$$
\begin{aligned}
q(\xi, \eta, \varrho, O, R, \varphi)= & \frac{1}{2} \min \{\mathrm{\varrho}(O \xi, O \eta)+\varphi(O \xi)+\varphi(O \eta), \mathrm{\varrho}(R \xi, R \eta) \\
& +\varphi(R \xi)+\varphi(R \eta)\}
\end{aligned}
$$

Let $\alpha: \mathscr{M} \times \mathscr{M} \longrightarrow[0,+\infty)$ be a mapping. Set

$\left(A_{s^{p}}\right)$ If $\left\{\xi_{n}\right\}$ is a sequence in $\mathscr{M}$ satisfying $R \xi_{n} \longrightarrow R \xi$ as $n \longrightarrow+\infty$, then there is a subsequence $\left\{R \xi_{n_{k}}\right\}$ of $\left\{R \xi_{n}\right\}$ with $\alpha\left(R \xi_{n_{k}}, R \xi\right) \geq s^{p}$ for $k \in \mathbb{N}$

$\left(B_{s^{p}}\right)$ For $x, y \in C(O, R)$, one can get the condition of $\alpha($ $R x, R y) \geq s^{p}$ and $\alpha(R x, R y) \geq s^{p}$

Theorem 14. Let $(\mathscr{M}, \mathrm{Q})$ be a complete rectangular $b$-metric space with coefficient $s \geq 1$. Let $O, R: \mathscr{M} \longrightarrow \mathscr{M}$ be given selfmappings satisfying $O(\mathscr{M}) \subset R(\mathscr{M})$ and $R(\mathscr{M})$ is closed. Assume that $\varphi: \mathscr{M} \longrightarrow[0,+\infty)$ is a lower semicontinuous mapping and $\alpha: \mathscr{M} \times \mathscr{M} \longrightarrow[0,+\infty)$. If

(i) $O$ is $R-\alpha_{s^{p}}$-admissible

(ii) $O$ is generalized $\left(R-\alpha_{s^{p}}, \theta, \varphi\right)$ contractive

(iii) there is $\xi_{0} \in \mathscr{M}$ satisfying $\alpha\left(R \xi_{0}, O \xi_{0}\right) \geq s^{p}$

(iv) properties $\left(A_{s^{p}}\right)$ and $\left(B_{s^{p}}\right)$ are fulfilled

(v) $\alpha$ satisfies transitive property, i.e., for $\xi, \eta, \zeta \in \mathscr{M}$

$$
\alpha(\xi, \eta) \geq s^{p} \text { and } \alpha(\eta, \zeta) \geq s^{p} \Rightarrow \alpha(\xi, \zeta) \geq s^{p},
$$

then $O$ and $R$ possess a unique point of coincidence. Furthermore, if $O$ and $R$ are weakly compatible, then $O$ and $R$ possess a unique common fixed point in $\mathscr{M}$.

Proof. It follows from condition (iii) that one can choose an $\xi_{0} \in \mathscr{M}$ with $\alpha\left(R \xi_{0}, O \xi_{0}\right) \geq s^{p}$. Define sequences $\left\{\xi_{n}\right\}$ and \{ $\left.\eta_{n}\right\}$ in $\mathscr{M}$ by $\eta_{n}=O \xi_{n}=R \xi_{n+1}$ for $n \in \mathbb{N}$. If $\eta_{n}=\eta_{n+1}$ for some $n$, then we deduce $\eta_{n}=\eta_{n+1}=O \xi_{n+1}=R \xi_{n+1}$ and $O$ and $R$ possess a point of coincidence. Next, we suppose that $\eta_{n} \neq \eta_{n+1}$ for $n \in \mathbb{N}$. In light of contraction condition (i), we 
obtain

$$
\begin{aligned}
& \alpha\left(R \xi_{0}, R \xi_{1}\right)=\alpha\left(R \xi_{0}, O \xi_{0}\right) \geq s^{p} \\
& \alpha\left(R \xi_{1}, R \xi_{2}\right)=\alpha\left(O \xi_{0}, O \xi_{1}\right) \geq s^{p} \\
& \alpha\left(R \xi_{2}, R \xi_{3}\right)=\alpha\left(O \xi_{1}, O \xi_{2}\right) \geq s^{p}
\end{aligned}
$$

Hence, for all $n \in \mathbb{N}$, we deduce $\alpha\left(R \xi_{n}, R \xi_{n+1}\right)=\alpha\left(\eta_{n-1}\right.$, $\left.\eta_{n}\right) \geq s^{p}$. Applying (14) with $\xi=\xi_{n}$ and $\eta=\xi_{n+1}$,

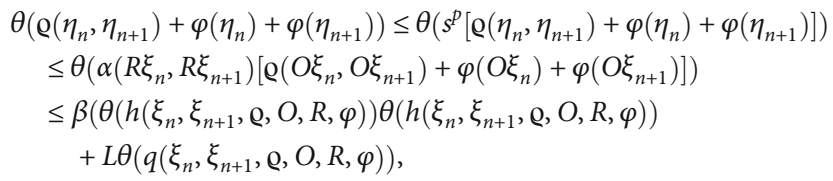

where

$$
\begin{aligned}
h\left(\xi_{n},\right. & \left.\xi_{n+1}, \mathrm{\varrho}, O, R, \varphi\right) \\
= & \max \left\{\mathrm{\varrho}\left(O \xi_{n}, O \xi_{n+1}\right)+\varphi\left(O \xi_{n}\right)+\varphi\left(O \xi_{n+1}\right), \frac{\mathrm{\varrho}\left(O \xi_{n+1}, R \xi_{n+1}\right)+\varphi\left(O \xi_{n+1}\right)+\varphi\left(R \xi_{n+1}\right)}{1+\mathrm{\varrho}\left(O \xi_{n}, R \xi_{n}\right)+\varphi\left(O \xi_{n}\right)+\varphi\left(R \xi_{n}\right)}\right. \\
& \cdot\left\{\mathrm{\varrho}\left(R \xi_{n}, R \xi_{n+1}\right)+\varphi\left(R \xi_{n}\right)+\varphi\left(R \xi_{n+1}\right)\right\}, \frac{1}{2}\left\{\mathrm{\varrho}\left(O \xi_{n}, O \xi_{n+1}\right)\right. \\
& \left.\left.+\varphi\left(O \xi_{n}\right)+\varphi\left(O \xi_{n+1}\right)+\mathrm{\varrho}\left(R \xi_{n}, R \xi_{n+1}\right)+\varphi\left(R \xi_{n}\right)+\varphi\left(R \xi_{n+1}\right)\right\}\right\} \\
= & \max \left\{\mathrm{\varrho}\left(\eta_{n}, \eta_{n+1}\right)+\varphi\left(\eta_{n}\right)+\varphi\left(\eta_{n+1}\right), \frac{\mathrm{\varrho}\left(\eta_{n+1}, \eta_{n}\right)+\varphi\left(\eta_{n+1}\right)+\varphi\left(\eta_{n}\right)}{1+\mathrm{\varrho}\left(\eta_{n}, \eta_{n-1}\right)+\varphi\left(\eta_{n}\right)+\varphi\left(\eta_{n-1}\right)}\right. \\
& \cdot\left\{\mathrm{\varrho}\left(\eta_{n-1}, \eta_{n}\right)+\varphi\left(\eta_{n-1}\right)+\varphi\left(\eta_{n}\right)\right\}, \frac{1}{2}\left\{\mathrm{\varrho}\left(\eta_{n}, \eta_{n+1}\right)+\varphi\left(\eta_{n}\right)\right. \\
& \left.\left.+\varphi\left(\eta_{n+1}\right)+\mathrm{\varrho}\left(\eta_{n-1}, \eta_{n}\right)+\varphi\left(\eta_{n-1}\right)+\varphi\left(\eta_{n}\right)\right\}\right\} \\
\leq & \max \left\{\mathrm{\varrho}\left(\eta_{n-1}, \eta_{n}\right)+\varphi\left(\eta_{n-1}\right)+\varphi\left(\eta_{n}\right), \mathrm{\varrho}\left(\eta_{n+1}, \eta_{n}\right)+\varphi\left(\eta_{n+1}\right)+\varphi\left(\eta_{n}\right)\right\},
\end{aligned}
$$

$$
\begin{aligned}
q\left(\xi_{n},\right. & \left.\xi_{n+1}, \varrho, O, R, \varphi\right)=\frac{1}{2} \min \left\{\mathrm{\varrho}\left(O \xi_{n}, O \xi_{n+1}\right)+\varphi\left(O \xi_{n}\right)\right. \\
& \left.+\varphi\left(O \xi_{n+1}\right), \varrho\left(R \xi_{n}, R \xi_{n+1}\right)+\varphi\left(R \xi_{n}\right)+\varphi\left(R \xi_{n+1}\right)\right\} \\
= & \frac{1}{2} \min \left\{\mathrm{\varrho}\left(\eta_{n}, \eta_{n+1}\right)+\varphi\left(\eta_{n}\right)+\varphi\left(\eta_{n+1}\right), \varrho\left(\eta_{n-1}, \eta_{n}\right)\right. \\
& \left.+\varphi\left(\eta_{n-1}\right)+\varphi\left(\eta_{n}\right)\right\} .
\end{aligned}
$$

If we assume that $\mathrm{Q}\left(\eta_{n}, \eta_{n+1}\right)+\varphi\left(\eta_{n}\right)+\varphi\left(\eta_{n+1}\right)>\mathrm{Q}\left(\eta_{n}\right.$, $\left.\eta_{n-1}\right)+\varphi\left(\eta_{n}\right)+\varphi\left(\eta_{n-1}\right)$ for some $n \in \mathbb{N}$, according to (18), (19), and (20), we have

$$
\begin{aligned}
& \theta\left(\mathrm{\varrho}\left(\eta_{n}, \eta_{n+1}\right)+\varphi\left(\eta_{n}\right)+\varphi\left(\eta_{n+1}\right)\right) \leq \frac{1}{s} \theta\left(h\left(\xi_{n}, \xi_{n+1}, \varrho, O, R, \varphi\right)\right) \\
& \quad+L \theta\left(q\left(\xi_{n}, \xi_{n+1}, \varrho, O, R, \varphi\right)\right) \\
& \leq \frac{1}{s} \theta\left(\mathrm{\varrho}\left(\eta_{n+1}, \eta_{n}\right)+\varphi\left(\eta_{n+1}\right)+\varphi\left(\eta_{n}\right)\right)+L \theta\left(\mathrm{\varrho}\left(\eta_{n+1}, \eta_{n}\right)\right. \\
&\left.\quad+\varphi\left(\eta_{n+1}\right)+\varphi\left(\eta_{n}\right)\right)<\theta\left(\mathrm{\varrho}\left(\eta_{n}, \eta_{n+1}\right)+\varphi\left(\eta_{n}\right)+\varphi\left(\eta_{n+1}\right)\right)
\end{aligned}
$$

which is a contradiction. Thus,

$$
\mathrm{Q}\left(\eta_{n}, \eta_{n+1}\right)+\varphi\left(\eta_{n}\right)+\varphi\left(\eta_{n+1}\right) \leq \mathrm{Q}\left(\eta_{n}, \eta_{n-1}\right)+\varphi\left(\eta_{n}\right)+\varphi\left(\eta_{n-1}\right),
$$

$$
\begin{aligned}
& h\left(\xi_{n}, \xi_{n+1}, \varrho, O, R, \varphi\right) \leq \mathrm{\varrho}\left(\eta_{n}, \eta_{n-1}\right)+\varphi\left(\eta_{n}\right)+\varphi\left(\eta_{n-1}\right), \\
& q\left(\xi_{n}, \xi_{n+1}, \varrho, O, R, \varphi\right)<\mathrm{\varrho}\left(\eta_{n}, \eta_{n-1}\right)+\varphi\left(\eta_{n}\right)+\varphi\left(\eta_{n-1}\right) .
\end{aligned}
$$

It follows from (22) that $\left\{\mathrm{\varrho}\left(\eta_{n}, \eta_{n+1}\right)+\varphi\left(\eta_{n}\right)+\varphi\left(\eta_{n+1}\right)\right\}$ is decreasing. It follows that there exists a real number $\gamma \geq 0$ satisfying

$$
\lim _{n \longrightarrow+\infty}\left(\mathrm{\varrho}\left(\eta_{n}, \eta_{n+1}\right)+\varphi\left(\eta_{n}\right)+\varphi\left(\eta_{n+1}\right)\right)=\gamma .
$$

In view of (18), (23), and (24), one can obtain

$$
\begin{aligned}
\theta\left(\mathrm{\varrho}\left(\eta_{n}, \eta_{n+1}\right)+\varphi\left(\eta_{n}\right)+\varphi\left(\eta_{n+1}\right)\right) \\
\leq \beta\left(\theta\left(h\left(\xi_{n}, \xi_{n+1}, \varrho, O, R, \varphi\right)\right) \theta\left(h\left(\xi_{n}, \xi_{n+1}, \varrho, O, R, \varphi\right)\right)\right. \\
\quad+L \theta\left(q\left(\xi_{n}, \xi_{n+1}, \varrho, O, R, \varphi\right)\right) \\
\quad<\theta\left(\mathrm{\varrho}\left(\eta_{n}, \eta_{n-1}\right)+\varphi\left(\eta_{n}\right)+\varphi\left(\eta_{n-1}\right)\right) .
\end{aligned}
$$

If $\gamma>0$, putting $n \longrightarrow \infty$ in (26), we obtain

a contradiction. Hence,

$$
\lim _{n \longrightarrow+\infty}\left(\mathrm{\varrho}\left(\eta_{n}, \eta_{n+1}\right)+\varphi\left(\eta_{n}\right)+\varphi\left(\eta_{n+1}\right)\right)=\gamma=0
$$

which implies that $\lim _{n \longrightarrow+\infty} \mathrm{\varrho}\left(\eta_{n}, \eta_{n+1}\right)=0$ and $\lim _{n \longrightarrow+\infty}$ $\varphi\left(\eta_{n}\right)=0$. In view of hypothesis (v), we have $\alpha\left(\eta_{n-2}, \eta_{n}\right) \geq$ $s^{p}$. Taking $\xi=\xi_{n-1}$ and $\eta=\xi_{n+1}$ in (14), we obtain

$$
\begin{aligned}
& \theta\left(\mathrm{\varrho}\left(\eta_{n-1}, \eta_{n+1}\right)+\varphi\left(\eta_{n-1}\right)+\varphi\left(\eta_{n+1}\right)\right) \\
& \leq \theta\left(\alpha\left(\eta_{n-2}, \eta_{n}\right)\left[\mathrm{\varrho}\left(\eta_{n-1}, \eta_{n+1}\right)+\varphi\left(\eta_{n-1}\right)+\varphi\left(\eta_{n+1}\right)\right]\right) \\
& \leq \beta\left(\theta\left(h\left(\xi_{n-1}, \xi_{n+1}, \varrho, O, R, \varphi\right)\right) \theta\left(h\left(\xi_{n-1}, \xi_{n+1}, \varrho, O, R, \varphi\right)\right)\right. \\
&+L \theta\left(q\left(\xi_{n-1}, \xi_{n+1}, \varrho, O, R, \varphi\right)\right),
\end{aligned}
$$

where

$$
\begin{aligned}
h\left(\xi_{n-1}, \xi_{n+1}, \varrho, O, R, \varphi\right)= & \max \left\{\mathrm{\varrho}\left(O \xi_{n-1}, O \xi_{n+1}\right)+\varphi\left(O \xi_{n-1}\right)+\varphi\left(O \xi_{n+1}\right),\right. \\
& \cdot \frac{\mathrm{\varrho}\left(O \xi_{n+1}, R \xi_{n+1}\right)+\varphi\left(O \xi_{n+1}\right)+\varphi\left(R \xi_{n+1}\right)}{1+\varrho\left(O \xi_{n-1}, R \xi_{n-1}\right)+\varphi\left(O \xi_{n-1}\right)+\varphi\left(R \xi_{n-1}\right)} \\
& \cdot\left\{\mathrm{\varrho}\left(R \xi_{n-1}, R \xi_{n+1}\right)+\varphi\left(R \xi_{n-1}\right)+\varphi\left(R \xi_{n+1}\right)\right\} \\
& \cdot \frac{1}{2}\left\{\mathrm{\varrho}\left(O \xi_{n-1}, O \xi_{n+1}\right)+\varphi\left(O \xi_{n-1}\right)+\varphi\left(O \xi_{n+1}\right)\right. \\
& \left.\left.+\mathrm{\varrho}\left(R \xi_{n-1}, R \xi_{n+1}\right)+\varphi\left(R \xi_{n-1}\right)+\varphi\left(R \xi_{n+1}\right)\right\}\right\} \\
\leq & \max \left\{\mathrm{\varrho}\left(\eta_{n-1}, \eta_{n+1}\right)+\varphi\left(\eta_{n-1}\right)+\varphi\left(\eta_{n+1}\right), \mathrm{\varrho}\left(\eta_{n-2}, \eta_{n}\right)\right. \\
& \left.+\varphi\left(\eta_{n-2}\right)+\varphi\left(\eta_{n}\right)\right\}
\end{aligned}
$$




$$
\begin{aligned}
q\left(\xi_{n-1}, \xi_{n+1}, \varrho, O, R, \varphi\right)= & \frac{1}{2} \min \left\{\mathrm{\varrho}\left(O \xi_{n-1}, O \xi_{n+1}\right)+\varphi\left(O \xi_{n-1}\right)\right. \\
& +\varphi\left(O \xi_{n+1}\right), \mathrm{\varrho}\left(R \xi_{n-1}, R \xi_{n+1}\right)+\varphi\left(R \xi_{n-1}\right) \\
& \left.+\varphi\left(R \xi_{n+1}\right)\right\} \\
= & \frac{1}{2} \min \left\{\mathrm{\varrho}\left(\eta_{n-1}, \eta_{n+1}\right)+\varphi\left(\eta_{n-1}\right)\right. \\
& \left.+\varphi\left(\eta_{n+1}\right), \varrho\left(\eta_{n-2}, \eta_{n}\right)+\varphi\left(\eta_{n-2}\right)+\varphi\left(\eta_{n}\right)\right\} .
\end{aligned}
$$

If for some $n \in \mathbb{N}, \varrho\left(\eta_{n-1}, \eta_{n+1}\right)+\varphi\left(\eta_{n-1}\right)+\varphi\left(\eta_{n+1}\right)>\mathrm{\varrho}($ $\left.\eta_{n-2}, \eta_{n}\right)+\varphi\left(\eta_{n-2}\right)+\varphi\left(\eta_{n}\right)$, according to (29), (30), and (31), we get

$$
\begin{aligned}
\theta & \left(\mathrm{\varrho}\left(\eta_{n-1}, \eta_{n+1}\right)+\varphi\left(\eta_{n-1}\right)+\varphi\left(\eta_{n+1}\right)\right) \\
& <\frac{1}{s} \theta\left(h\left(\xi_{n-1}, \xi_{n+1}, \varrho, O, R, \varphi\right)\right)+L \theta\left(q\left(\xi_{n-1}, \xi_{n+1}, \varrho, O, R, \varphi\right)\right) \\
& <\theta\left(\mathrm{\varrho}\left(\eta_{n-1}, \eta_{n+1}\right)+\varphi\left(\eta_{n-1}\right)+\varphi\left(\eta_{n+1}\right)\right),
\end{aligned}
$$

which is a contradiction. It follows that

$$
\mathrm{\varrho}\left(\eta_{n-1}, \eta_{n+1}\right)+\varphi\left(\eta_{n-1}\right)+\varphi\left(\eta_{n+1}\right) \leq \mathrm{\varrho}\left(\eta_{n-2}, \eta_{n}\right)+\varphi\left(\eta_{n-2}\right)+\varphi\left(\eta_{n}\right),
$$

$$
h\left(\xi_{n-1}, \xi_{n+1}, \varrho, O, R, \varphi\right) \leq \varrho\left(\eta_{n-2}, \eta_{n}\right)+\varphi\left(\eta_{n-2}\right)+\varphi\left(\eta_{n}\right),
$$

$$
q\left(\xi_{n-1}, \xi_{n+1}, \varrho, O, R, \varphi\right)<\varrho\left(\eta_{n-2}, \eta_{n}\right)+\varphi\left(\eta_{n-2}\right)+\varphi\left(\eta_{n}\right) .
$$

Inequality (33) yields that $\left\{\mathrm{Q}\left(\eta_{n-2}, \eta_{n}\right)+\varphi\left(\eta_{n-2}\right)+\varphi\left(\eta_{n}\right.\right.$ )$\}$ is non-increasing and which yields that there exists $\in \geq$ 0 satisfying

$$
\lim _{n \longrightarrow+\infty}\left(\mathrm{\varrho}\left(\eta_{n-2}, \eta_{n}\right)+\varphi\left(\eta_{n-2}\right)+\varphi\left(\eta_{n}\right)\right)=\epsilon
$$

In light of (32), (34), and (35), one can deduce

$$
\begin{aligned}
\theta\left(\mathrm{\varrho}\left(\eta_{n-1}, \eta_{n+1}\right)+\varphi\left(\eta_{n-1}\right)+\varphi\left(\eta_{n+1}\right)\right) \\
\leq \beta\left(\theta\left(h\left(\xi_{n-1}, \xi_{n+1}, \varrho, \mathrm{O}, R, \varphi\right)\right) \theta\left(h\left(\xi_{n-1}, \xi_{n+1}, \varrho, O, R, \varphi\right)\right)\right. \\
\quad+L \theta\left(q\left(\xi_{n-1}, \xi_{n+1}, \varrho, O, R, \varphi\right)\right) \\
\quad<\theta\left(\mathrm{\varrho}\left(\eta_{n-2}, \eta_{n}\right)+\varphi\left(\eta_{n-2}\right)+\varphi\left(\eta_{n}\right)\right) .
\end{aligned}
$$

Assume that $\epsilon>0$. Letting $n \longrightarrow \infty$ in (37), we derive

$$
\begin{aligned}
\theta(\epsilon)= & \lim _{n \longrightarrow+\infty} \theta\left(\varrho\left(\eta_{n-1}, \eta_{n+1}\right)+\varphi\left(\eta_{n-1}\right)+\varphi\left(\eta_{n+1}\right)\right) \\
\leq & \lim _{n \longrightarrow+\infty} \beta\left(\theta\left(h\left(\xi_{n-1}, \xi_{n+1}, \varrho, O, R, \varphi\right)\right) \theta\left(h\left(\xi_{n-1}, \xi_{n+1}, \varrho, O, R, \varphi\right)\right)\right. \\
& \left.+L \lim _{n \longrightarrow+\infty} \theta\left(q\left(\xi_{n-1}, \xi_{n+1}, \varrho, O, R, \varphi\right)\right)\right) \\
< & \lim _{n \longrightarrow+\infty} \theta\left(\varrho\left(\eta_{n-2}, \eta_{n}\right)+\varphi\left(\eta_{n-2}\right)+\varphi\left(\eta_{n}\right)\right)=\theta(\epsilon),
\end{aligned}
$$

which gives a contradiction. This yields that

$$
\lim _{n \longrightarrow+\infty}\left(\varrho\left(\eta_{n-2}, \eta_{n}\right)+\varphi\left(\eta_{n-2}\right)+\varphi\left(\eta_{n}\right)\right)=\epsilon=0 .
$$

It follows that $\lim _{n \longrightarrow+\infty} \mathrm{Q}\left(\eta_{n-2}, \eta_{n}\right)=0$.

Now, we aim to show that $\left\{\eta_{n}\right\}$ is a Cauchy sequence. Assume on the contrary that, $\left\{\eta_{n}\right\}$ is not Cauchy. So, there exists $\varepsilon>0$ for which we can choose sequences $\left\{\eta_{m_{k}}\right\}$ and $\left\{\eta_{n_{k}}\right\}$ of $\left\{\eta_{n}\right\}$ such that $n_{k}$ is the smallest index for which $n_{k}>m_{k}>k$,

$$
\begin{gathered}
\varepsilon \leq \mathrm{Q}\left(\eta_{m_{k}}, \eta_{n_{k}}\right), \\
\varrho\left(\eta_{m_{k}}, \eta_{n_{k}-1}\right)<\varepsilon .
\end{gathered}
$$

In light of the rectangular inequality and (40) and (41), we have

$$
\begin{aligned}
\varepsilon & \leq \mathrm{Q}\left(\eta_{m_{k}}, \eta_{n_{k}}\right) \leq s\left[\mathrm{Q}\left(\eta_{m_{k}}, \eta_{n_{k}-1}\right)+\mathrm{Q}\left(\eta_{n_{k}-1}, \eta_{n_{k}+1}\right)+\mathrm{Q}\left(\eta_{n_{k}+1}, \eta_{n_{k}}\right)\right] \\
& <s \varepsilon+s \mathrm{Q}\left(\eta_{n_{k}-1}, \eta_{n_{k}+1}\right)+s \mathrm{Q}\left(\eta_{n_{k}+1}, \eta_{n_{k}}\right) .
\end{aligned}
$$

Taking the superior limit as $k \longrightarrow+\infty$, we have

$$
\varepsilon \leq \limsup _{k \longrightarrow+\infty} \varrho\left(\eta_{m_{k}}, \eta_{n_{k}}\right) \leq s \varepsilon
$$

Similarly,

$\mathrm{\varrho}\left(\eta_{m_{k}}, \eta_{n_{k}}\right) \leq s\left[\mathrm{\varrho}\left(\eta_{m_{k}}, \eta_{m_{k}+1}\right)+\mathrm{\varrho}\left(\eta_{m_{k}+1}, \eta_{m_{k}-1}\right)+\mathrm{\varrho}\left(\eta_{m_{k}-1}, \eta_{n_{k}}\right)\right]$,

$\mathrm{\varrho}\left(\eta_{m_{k}}, \eta_{n_{k}}\right) \leq s\left[\mathrm{\varrho}\left(\eta_{m_{k}}, \eta_{m_{k}-1}\right)+\mathrm{\varrho}\left(\eta_{m_{k}-1}, \eta_{n_{k}-1}\right)+\mathrm{\varrho}\left(\eta_{n_{k}-1}, \eta_{n_{k}}\right)\right]$,

$\mathrm{\varrho}\left(\eta_{m_{k}-1}, \eta_{n_{k}}\right) \leq s\left[\mathrm{\varrho}\left(\eta_{m_{k}-1}, \eta_{m_{k}}\right)+\mathrm{\varrho}\left(\eta_{m_{k}}, \eta_{n_{k}-1}\right)+\mathrm{\varrho}\left(\eta_{n_{k}-1}, \eta_{n_{k}}\right)\right]$.

It follows from (40), (41), and (42) that

$$
\frac{\varepsilon}{s} \leq \limsup _{k \longrightarrow+\infty}\left(\eta_{m_{k}}, \eta_{n_{k}-1}\right) \leq \varepsilon
$$

By (40), (41), (44), and (46), we get

$$
\frac{\varepsilon}{s} \leq \limsup _{k \longrightarrow+\infty}\left(\eta_{m_{k}-1}, \eta_{n_{k}}\right) \leq s \varepsilon
$$

By the similar method, we have

$$
\mathrm{\varrho}\left(\eta_{m_{k}-1}, \eta_{n_{k}-1}\right) \leq s\left[\mathrm{\varrho}\left(\eta_{m_{k}-1}, \eta_{m_{k}}\right)+\mathrm{\varrho}\left(\eta_{m_{k}}, \eta_{n_{k}}\right)+\mathrm{\varrho}\left(\eta_{n_{k}}, \eta_{n_{k}-1}\right)\right],
$$


so is

$$
\frac{\varepsilon}{s} \leq \limsup _{k \longrightarrow+\infty}\left(\eta_{m_{k}-1}, \eta_{n_{k}-1}\right) \leq s^{2} \varepsilon
$$

According to the definition of $h(\xi, \eta, \varrho, O, R, \varphi)$, we get

$$
\begin{aligned}
& h\left(\xi_{m_{k}}, \xi_{n_{k}}, \mathrm{\varrho}, O, R, \varphi\right)=\max \left\{\mathrm{\varrho}\left(O \xi_{m_{k}}, O \xi_{n_{k}}\right)+\varphi\left(O \xi_{m_{k}}\right)\right. \\
& \quad+\varphi\left(O \xi_{n_{k}}\right), \frac{\mathrm{\varrho}\left(O \xi_{n_{k}}, R \xi_{n_{k}}\right)+\varphi\left(O \xi_{n_{k}}\right)+\varphi\left(R \xi_{n_{k}}\right)}{1+\rho\left(O \xi_{m_{k}}, R \xi_{m_{k}}\right)+\varphi\left(O \xi_{m_{k}}\right)+\varphi\left(R \xi_{m_{k}}\right)} \\
& \quad \cdot\left\{\mathrm{\varrho}\left(R \xi_{m_{k}}, R \xi_{n_{k}}\right)+\varphi\left(R \xi_{m_{k}}\right)+\varphi\left(R \xi_{n_{k}}\right)\right\}, \frac{1}{2}\left\{\mathrm{\varrho}\left(O \xi_{m_{k}}, O \xi_{n_{k}}\right)+\varphi\left(O \xi_{m_{k}}\right)\right. \\
& \left.\left.\quad+\varphi\left(O \xi_{n_{k}}\right)+\mathrm{\varrho}\left(R \xi_{m_{k}}, R \xi_{n_{k}}\right)+\varphi\left(R \xi_{m_{k}}\right)+\varphi\left(R \xi_{n_{k}}\right)\right\}\right\} \\
& =\max \left\{\mathrm{\varrho}\left(\eta_{m_{k}}, \eta_{n_{k}}\right)+\varphi\left(\eta_{m_{k}}\right)+\varphi\left(\eta_{n_{k}}\right), \frac{\mathrm{\varrho}\left(\eta_{n_{k}}, \eta_{n_{k}-1}\right)+\varphi\left(\eta_{n_{k}}\right)+\varphi\left(\eta_{n_{k}-1}\right)}{1+\mathrm{\varrho}\left(\eta_{m_{k}}, \eta_{m_{k}-1}\right)+\varphi\left(\eta_{m_{k}}\right)+\varphi\left(\eta_{m_{k}-1}\right)}\right. \\
& \quad \cdot\left\{\mathrm{\varrho}\left(\eta_{m_{k}-1}, \eta_{n_{k}-1}\right)+\varphi\left(\eta_{m_{k}-1}\right)+\varphi\left(\eta_{n_{k}-1}\right)\right\}, \frac{1}{2}\left\{\mathrm{\varrho}\left(\eta_{m_{k}}, \eta_{n_{k}}\right)+\varphi\left(\eta_{m_{k}}\right)+\varphi\left(\eta_{n_{k}}\right)\right. \\
& \left.\left.\quad+\mathrm{\varrho}\left(\eta_{m_{k}-1}, \eta_{n_{k}-1}\right)+\varphi\left(\eta_{m_{k}-1}\right)+\varphi\left(\eta_{n_{k}-1}\right)\right\}\right\} .
\end{aligned}
$$

Taking the superior limit as $k \longrightarrow+\infty$ in (51), we get

$$
\limsup _{k \longrightarrow+\infty} h\left(\xi_{m_{k}}, \xi_{n_{k}}, \varrho, O, R, \varphi\right) \leq \max \left\{s \varepsilon, 0, \frac{s \varepsilon+s^{2} \varepsilon}{2}\right\}<s^{2} \varepsilon
$$

Also, we have

$$
\begin{aligned}
q\left(\xi_{m_{k}}, \xi_{n_{k}}, \mathrm{\varrho}, O, R, \varphi\right)= & \frac{1}{2} \min \left\{\mathrm{\varrho}\left(O \xi_{m_{k}}, O \xi_{n_{k}}\right)+\varphi\left(O \xi_{m_{k}}\right)+\varphi\left(O \xi_{n_{k}}\right), \mathrm{\varrho}\left(R \xi_{m_{k}}, R \xi_{n_{k}}\right)\right. \\
& \left.+\varphi\left(R \xi_{m_{k}}\right)+\varphi\left(R \xi_{n_{k}}\right)\right\} \\
= & \frac{1}{2} \min \left\{\mathrm{\varrho}\left(\eta_{m_{k}}, \eta_{n_{k}}\right)+\varphi\left(\eta_{m_{k}}\right)+\varphi\left(\eta_{n_{k}}\right), \varrho\left(\eta_{m_{k}-1}, \eta_{n_{k}-1}\right)\right. \\
& \left.+\varphi\left(\eta_{m_{k}-1}\right)+\varphi\left(\eta_{n_{k}-1}\right)\right\} .
\end{aligned}
$$

It follows that

$$
\limsup _{k \longrightarrow+\infty} q\left(\xi_{m_{k}}, \xi_{n_{k}}, \varrho, O, R, \varphi\right)<s^{2} \varepsilon
$$

The transitivity property of $\alpha$ yields that $\alpha\left(R \xi_{m_{k}}, R \xi_{n_{k}}\right)$ $\geq s^{p}$. Taking $\xi=\xi_{m_{k}}$ and $\eta=\xi_{n_{k}}$ in (14), one can deduce

$$
\begin{aligned}
\theta\left(s^{2} \varepsilon\right) \leq & \theta\left(s^{p} \varepsilon\right) \leq \theta\left(\alpha\left(R \xi_{m_{k}}, R \xi_{n_{k}}\right) \limsup _{n \longrightarrow+\infty}\left[\mathrm{\varrho}\left(\eta_{m_{k}}, \eta_{n_{k}}\right)+\varphi\left(\eta_{m_{k}}\right)+\varphi\left(\eta_{n_{k}}\right)\right]\right) \\
\leq & \limsup _{n \longrightarrow+\infty} \beta\left(\theta\left(h\left(\xi_{m_{k}}, \xi_{n_{k}}, \varrho, O, R, \varphi\right)\right)\right) \theta\left(h\left(\xi_{m_{k}}, \xi_{n_{k}}, \varrho, O, R, \varphi\right)\right) \\
& +\limsup _{n \longrightarrow+\infty} L \theta\left(q\left(\xi_{m_{k}}, \xi_{n_{k}}, \varrho, O, R, \varphi\right)\right) \\
\leq & \frac{1}{s} \theta\left(s^{2} \varepsilon\right)+L \theta\left(s^{2} \varepsilon\right)<\theta\left(s^{2} \varepsilon\right),
\end{aligned}
$$

a contradiction. Hence, $\left\{\eta_{n}\right\}$ is Cauchy. Since $(\mathscr{M}, \rho)$ is complete, there is a $\vartheta \in \mathscr{M}$ such that

$$
\lim _{n \longrightarrow+\infty} \mathrm{\varrho}\left(\eta_{n}, 9\right)=\lim _{n \longrightarrow+\infty} \mathrm{\varrho}\left(O \xi_{n}, 9\right)=\lim _{n \longrightarrow+\infty} \mathrm{\varrho}\left(R \xi_{n+1}, \vartheta\right)=\lim _{n, m \longrightarrow+\infty} \mathrm{\varrho}\left(\eta_{n}, \eta_{m}\right)=0 .
$$

Since $R(\mathscr{M})$ is closed, we have $\vartheta \in R(\mathscr{M})$. Hence,we choose a $z \in \mathscr{M}$ satisfying $\vartheta=R z$. We write (56) as

$$
\lim _{n \longrightarrow+\infty} \mathrm{Q}\left(\eta_{n}, R z\right)=\lim _{n \longrightarrow+\infty} \mathrm{\varrho}\left(O \xi_{n}, R z\right)=\lim _{n \longrightarrow+\infty} \mathrm{\varrho}\left(R \xi_{n+1}, R z\right)=0 .
$$

It follows from the definition of $\varphi$ that

$$
\varphi(R z)=\varphi(\vartheta) \leq \liminf _{n \longrightarrow+\infty} \varphi\left(\eta_{n}\right)=0,
$$

which implies that $\varphi(R z)=\varphi(\vartheta)=0$.

The property $\left(A_{s^{p}}\right)$ ensures that there exists a subsequence $\left\{\eta_{n_{k}}\right\}$ of $\left\{\eta_{n}\right\}$ with $\alpha\left(\eta_{n_{k}-1}, R z\right) \geq s^{p}$ for $k \in \mathbb{N}$. If $\mathrm{Q}($ $O z, R z)+\varphi(O z) \neq 0$, taking $\xi=\xi_{\mathrm{n}_{k}}$ and $\eta=z$ in (14), one deduce that

$$
\begin{aligned}
& \theta\left(\varrho\left(O \xi_{n_{k}}, O z\right)+\varphi\left(O \xi_{n_{k}}\right)+\varphi(O z)\right) \\
& \leq \theta\left(s^{p}\left[\varrho\left(O \xi_{n_{k}}, O z\right)+\varphi\left(O \xi_{n_{k}}\right)+\varphi(O z)\right]\right) \\
& \leq \theta\left(\alpha\left(\eta_{n_{k}-1}, R z\right)\left[\varrho\left(O \xi_{n_{k}}, O z\right)+\varphi\left(O \xi_{n_{k}}\right)+\varphi(O z)\right]\right) \\
& \leq \beta\left(\theta\left(h\left(\xi_{n_{k}}, z, \varrho, O, R, \varphi\right)\right)\right) \theta\left(h\left(\xi_{n_{k}}, z, \varrho, O, R, \varphi\right)\right) \\
& \quad+L \theta\left(q\left(\xi_{n_{k}}, z, \varrho, O, R, \varphi\right)\right),
\end{aligned}
$$

where

$$
\begin{aligned}
h\left(\xi_{n_{k}}, z, \varrho, O, R, \varphi\right)= & \max \left\{\mathrm{\varrho}\left(O \xi_{n_{k}}, O z\right)+\varphi\left(O \xi_{n_{k}}\right)+\varphi(O z),\right. \\
& \cdot \frac{\mathrm{\varrho}(O z, R z)+\varphi(O z)+\varphi(R z)}{1+\rho\left(O \xi_{n_{k}}, R \xi_{n_{k}}\right)+\varphi\left(O \xi_{n_{k}}\right)+\varphi\left(R \xi_{n_{k}}\right)} \\
& \cdot\left\{\mathrm{\varrho}\left(R \xi_{n_{k}}, R z\right)+\varphi\left(R \xi_{n_{k}}\right)+\varphi(R z)\right\}, \\
& \cdot \frac{1}{2}\left\{\mathrm{\varrho}\left(O \xi_{n_{k}}, O z\right)+\varphi\left(O \xi_{n_{k}}\right)+\varphi(O z)\right. \\
& \left.\left.+\mathrm{\varrho}\left(R z, R \xi_{n_{k}}\right)+\varphi(R z)+\varphi\left(R \xi_{n_{k}}\right)\right\}\right\} \\
= & \max \left\{\mathrm{\varrho}\left(\eta_{n_{k}}, O z\right)+\varphi\left(\eta_{n_{k}}\right)+\varphi(O z),\right. \\
& \cdot \frac{\mathrm{\varrho}(O z, R z)+\varphi(O z)+\varphi(R z)}{1+\mathrm{\varrho}\left(\eta_{n_{k}}, \eta_{n_{k}-1}\right)+\varphi\left(\eta_{n_{k}}\right)+\varphi\left(\eta_{n_{k}-1}\right)} \\
& \cdot\left\{\mathrm{\varrho}\left(\eta_{n_{k}-1}, R z\right)+\varphi\left(\eta_{n_{k}-1}\right)+\varphi(R z)\right\}, \\
& \cdot \frac{1}{2}\left\{\mathrm{\varrho}\left(\eta_{n_{k}}, O z\right)+\varphi\left(\eta_{n_{k}}\right)+\varphi(O z)+\mathrm{\varrho}\left(\eta_{n_{k}-1}, R z\right)\right. \\
& \left.\left.+\varphi\left(\eta_{n_{k}-1}\right)+\varphi(R z)\right\}\right\},
\end{aligned}
$$




$$
\begin{aligned}
q\left(\xi_{n_{k}}, z, \mathrm{\varrho}, O, R, \varphi\right)= & \frac{1}{2} \min \left\{\mathrm{\varrho}\left(O \xi_{n_{k}}, O z\right)+\varphi\left(O \xi_{n_{k}}\right)\right. \\
& \left.+\varphi(O z), \mathrm{\varrho}\left(R \xi_{n_{k}}, R z\right)+\varphi\left(R \xi_{n_{k}}\right)+\varphi(R z)\right\} \\
= & \frac{1}{2} \min \left\{\mathrm{\varrho}\left(\eta_{n_{k}}, O z\right)+\varphi\left(\eta_{n_{k}}\right)+\varphi(O z), \mathrm{\varrho}\left(\eta_{n_{k}-1}, R z\right)\right. \\
& \left.+\varphi\left(\eta_{n_{k}-1}\right)+\varphi(R z)\right\} .
\end{aligned}
$$

By simple calculation, we get

$$
\underset{k \longrightarrow+\infty}{\lim \sup } h\left(\xi_{n_{k}}, z, \varrho, O, R, \varphi\right) \leq s(\mathrm{\varrho}(O z, R z)+\varphi(O z))
$$

$$
\limsup _{k \longrightarrow+\infty} q\left(\xi_{n_{k}}, z, \varrho, O, R, \varphi\right)<s(\rho(O z, R z)+\varphi(O z)) .
$$

Letting $k \longrightarrow+\infty$ in (59), using (62) and (63), we obtain

$$
\begin{aligned}
\theta(s(\mathrm{\varrho}(O z, R z)+\varphi(O z))) & \leq \theta\left(s^{2} \limsup _{k \rightarrow \infty}\left(\mathrm{\varrho}\left(O \xi_{n_{k}}, O z\right)+\varphi\left(O \xi_{n_{k}}\right)+\varphi(O z)\right)\right) \\
& \leq \theta\left(\limsup _{k \rightarrow \infty}\left(\alpha\left(\eta_{n_{k-1}}, R z\right)\left[\mathrm{\varrho}\left(O \xi_{n_{k}}, O z\right)+\varphi\left(O \xi_{n_{k}}\right)+\varphi(O z)\right]\right)\right. \\
& <\frac{1}{s} \theta(s(\mathrm{\varrho}(O z, R z)+\varphi(O z)))+L \theta(s(\mathrm{\varrho}(O z, R z)+\varphi(O z))) \\
& <\theta(s(\mathrm{\varrho}(O z, R z)+\varphi(O z))) .
\end{aligned}
$$

It follows that $\mathrm{Q}(\mathrm{Oz}, \mathrm{Rz})+\varphi(\mathrm{Oz})=0$, which implies that $\mathrm{O} z=\mathrm{R} z, \varphi(\mathrm{O} z)=0$.

Next, we show that $O$ and $R$ possess the unique point of coincidence 9 . Assume on the contrary, there exist $z, z^{\prime} \in C$ $(\mathrm{O}, R)$ and $O z \neq O z^{\prime}$. By the property of $\left(B_{s^{p}}\right)$, one can obtain

$$
\alpha\left(R z^{\prime}, R z\right) \geq s^{p}
$$

Taking $\xi=z^{\prime}$ and $\eta=z$ in (14), we obtain

$$
\begin{aligned}
& \theta\left(\varrho\left(O z^{\prime}, O z\right)+\varphi\left(O z^{\prime}\right)+\varphi(O z)\right) \\
& \leq \theta\left(s^{p}\left[\mathrm{\varrho}\left(O z^{\prime}, O z\right)+\varphi\left(O z^{\prime}\right)+\varphi(O z)\right]\right) \\
& \leq \theta\left(\alpha\left(R z^{\prime}, R z\right)\left[\varrho\left(O z^{\prime}, O z\right)+\varphi\left(O z^{\prime}\right)+\varphi(O z)\right]\right) \\
& \leq \beta\left(\theta\left(h\left(z^{\prime}, z, \varrho, O, R, \varphi\right)\right)\right) \theta\left(h\left(z^{\prime}, z, \varrho, O, R, \varphi\right)\right) \\
& \quad+L \theta\left(q\left(z^{\prime}, z, \varrho, O, R, \varphi\right)\right),
\end{aligned}
$$

where

$$
\begin{aligned}
h\left(z^{\prime}, z, \mathrm{\varrho}, O, R, \varphi\right)= & \max \left\{\mathrm{\varrho}\left(O z^{\prime}, O z\right)+\varphi\left(O z^{\prime}\right)+\varphi(O z),\right. \\
& \cdot \frac{\mathrm{\varrho}(O z, R z)+\varphi(O z)+\varphi(R z)}{1+\mathrm{\varrho}\left(O z^{\prime}, R z^{\prime}\right)+\varphi\left(O z^{\prime}\right)+\varphi\left(R z^{\prime}\right)} \\
& \cdot\left\{\mathrm{\varrho}\left(R z^{\prime}, R z\right)+\varphi\left(R z^{\prime}\right)+\varphi(R z)\right\}, \\
& \cdot \frac{1}{2}\left\{\mathrm{\varrho}\left(O z^{\prime}, O z\right)+\varphi\left(O z^{\prime}\right)+\varphi(O z)\right. \\
& \left.\left.+\mathrm{\varrho}\left(R z^{\prime}, R z\right)+\varphi\left(R z^{\prime}\right)+\varphi(R z)\right\}\right\} \\
\leq & \mathrm{\varrho}\left(R z^{\prime}, R z\right)+\varphi\left(R z^{\prime}\right), \\
q\left(z^{\prime}, z, \varrho, O, R, \varphi\right)= & \frac{1}{2} \min \left\{\mathrm{\varrho}\left(O z^{\prime}, O z\right)+\varphi\left(O z^{\prime}\right)\right. \\
& \left.+\varphi(O z), \varrho\left(R z^{\prime}, R z\right)+\varphi\left(R z^{\prime}\right)+\varphi(R z)\right\} \\
& <\mathrm{\varrho}\left(R z^{\prime}, R z\right)+\varphi\left(R z^{\prime}\right) .
\end{aligned}
$$

In view of (66), we have

$$
\begin{aligned}
\theta\left(\mathrm{\varrho}\left(R z^{\prime}, R z\right)+\varphi\left(R z^{\prime}\right)\right)< & \frac{1}{s} \theta\left(\mathrm{\varrho}\left(R z^{\prime}, R z\right)+\varphi\left(R z^{\prime}\right)\right) \\
& +L \theta\left(\mathrm{\varrho}\left(R z^{\prime}, R z\right)+\varphi\left(R z^{\prime}\right)\right) \\
< & \theta\left(\mathrm{\varrho}\left(R z^{\prime}, R z\right)+\varphi\left(R z^{\prime}\right)\right) .
\end{aligned}
$$

Therefore, one can obtain that $\mathrm{Q}\left(R z, R z^{\prime}\right)+\varphi\left(R z^{\prime}\right)=0$, that is, $R z=R z^{\prime}=\vartheta$ and $\varphi\left(R z^{\prime}\right)=0$. Hence, $\vartheta$ is a unique point of coincidence for $O$ and $R$. Furthermore, if $O$ and $R$ are weak compatible mappings, it is easy to prove that $O$ and $R$ have a unique common fixed point $z$. The proof is complete.

Example 3. Let $(\mathscr{M}, \mathrm{Q})$ be the same as it is in Example 1 . Define mappings $O, R: \mathscr{M} \longrightarrow \mathscr{M}$ by

$$
\begin{gathered}
O \xi=\left\{\begin{aligned}
\frac{1}{5}, & \xi \in A, \\
\frac{1}{3}, & \xi \in B,
\end{aligned}\right. \\
R \xi= \begin{cases}\frac{1}{5}, \quad \xi=\frac{1}{5}, \\
\frac{1}{3}, \quad \xi=\frac{1}{4}, \\
\frac{1}{2}, \quad \xi=\frac{1}{3}, \\
1, \quad \xi \in\left\{\frac{1}{2}\right\} \cup B .\end{cases}
\end{gathered}
$$


Define mappings $\alpha: \mathscr{M} \times \mathscr{M} \longrightarrow[0,+\infty)$ by

$\alpha(\xi, \eta)=\left\{\begin{array}{l}s^{p}, \xi, \eta \in\left\{\frac{1}{5}, \frac{1}{4}, \frac{1}{3}, \frac{1}{2}\right\} \text { with } \xi \neq \eta \text {, or } \xi=\eta=\frac{1}{5}, \text { or } \xi=\eta=1 \text {, or } \xi=\eta=\frac{1}{3}, \\ 0, \text { otherwise. }\end{array}\right.$ lows:

Define $\theta:[0,+\infty) \longrightarrow[0,+\infty), \varphi: \mathscr{M} \longrightarrow[0,+\infty)$ as fol-

$$
\varphi(\xi)= \begin{cases}0, \quad \xi \in\left[0, \frac{1}{5}\right], \\ 0.15 \xi-0.03, \quad \xi \in\left(\frac{1}{5}, \frac{1}{3}\right], \\ 11.97 \xi-3.97, \quad \xi \in\left(\frac{1}{3},+\infty\right) .\end{cases}
$$

Defined $\beta(\xi)=1 / 5$ for all $\xi \geq 0$, then $\beta \in \Omega$. We can show that $O(\mathscr{M}) \subset R(\mathscr{M}), R(\mathscr{M})$ is closed. For $\xi, \eta \in \mathscr{M}$ such that $\alpha(R \xi, R \eta) \geq s^{p}$, we get that $R \xi, R \eta \in\{1 / 5,1 / 4,1 / 3,1 / 2\}$ with $R \xi \neq R \eta$, or $R \xi=R \eta=1 / 5$, or $R \xi=R \eta=1$. This implies that $\xi, \eta \in\{1 / 5,1 / 4,1 / 3\}$ with $\xi \neq \eta$, or $\xi=\eta=1 / 5$, or $\xi=\eta=1 /$ 4 , or $\xi, \eta \in\{1 / 2\} \cup B$. So we obtain $O \xi, O \eta \in\{1 / 5,1 / 3\}$ and $\alpha(O \xi, O \eta) \geq s^{p}$. Combining with the condition $\mathrm{Q}(O \xi, O \eta)+$ $\varphi(O \xi)+\varphi(O \eta) \neq 0$, the following cases are considered:

Case 1. $\xi=1 / 2, \eta \in B($ or $\eta=1 / 2, \xi \in B)$.

$$
\begin{aligned}
& \theta(\alpha(R \xi, R \eta)[\varrho(O \xi, O \eta)+\varphi(O \xi)+\varphi(O \eta)]) \\
& \quad=\frac{1}{2} \cdot 16 \cdot\left[\varrho\left(\frac{1}{5}, \frac{1}{3}\right)+\varphi\left(\frac{1}{5}\right)+\varphi\left(\frac{1}{3}\right)\right]=0.8,
\end{aligned}
$$

$$
\begin{aligned}
\beta & \theta(h(\xi, \eta, \varrho, O, R, \varphi))) \theta(h(\xi, \eta, \varrho, O, R, \varphi))+L \theta(q(\xi, \eta, \varrho, O, R, \varphi)) \\
& \geq \beta(\theta(h(\xi, \eta, \varrho, O, R, \varphi))) \theta(h(\xi, \eta, \varrho, O, R, \varphi)) \\
& \geq \frac{1}{5} \cdot \frac{1}{2} \cdot \frac{1}{2}\left\{\varrho\left(\frac{1}{5}, \frac{1}{3}\right)+\varphi\left(\frac{1}{5}\right)+\varphi\left(\frac{1}{3}\right)+\varrho(1,1)+\varphi(1)+\varphi(1)\right\} \\
& =\frac{1}{20} \cdot(0.1+8+8)>0.8 .
\end{aligned}
$$

In view of above inequalities, one can get that

$$
\begin{aligned}
& \theta(\alpha(R \xi, R \eta)[\mathrm{\varrho}(O \xi, O \eta)+\varphi(O \xi)+\varphi(O \eta)]) \\
& \quad \leq \beta(\theta(h(\xi, \eta, \varrho, O, R, \varphi))) \theta(h(\xi, \eta, \varrho, O, R, \varphi))+L \theta(q(\xi, \eta, \varrho, O, R, \varphi)),
\end{aligned}
$$

with $L \geq 0,1 / s+L<1$ and $s=4, p=2$.
Case 2. $\xi, \eta \in B$.

$$
\begin{aligned}
& \theta(\alpha(R \xi, R \eta)[\mathrm{\varrho}(O \xi, O \eta)+\varphi(O \xi)+\varphi(O \eta)]) \\
& \quad=\frac{1}{2} \cdot 16 \cdot\left[\mathrm{\varrho}\left(\frac{1}{3}, \frac{1}{3}\right)+\varphi\left(\frac{1}{3}\right)+\varphi\left(\frac{1}{3}\right)\right]=0.32
\end{aligned}
$$

$$
\begin{aligned}
\beta( & \theta(h(\xi, \eta, \varrho, O, R, \varphi))) \theta(h(\xi, \eta, \varrho, O, R, \varphi))+L \theta(q(\xi, \eta, \varrho, O, R, \varphi)) \\
& \geq \beta(\theta(h(\xi, \eta, \varrho, O, R, \varphi))) \theta(h(\xi, \eta, \varrho, O, R, \varphi)) \\
& \geq \frac{1}{5} \cdot \frac{1}{2} \cdot \frac{1}{2}\left\{\varrho\left(\frac{1}{3}, \frac{1}{3}\right)+\varphi\left(\frac{1}{3}\right)+\varphi\left(\frac{1}{3}\right)+\varrho(1,1)+\varphi(1)+\varphi(1)\right\} \\
& =\frac{1}{20} \cdot(0.04+8+8)>0.32 .
\end{aligned}
$$

That is, for $\xi, \eta \in B$,

$$
\begin{aligned}
& \theta(\alpha(R \xi, R \eta)[\varrho(O \xi, O \eta)+\varphi(O \xi)+\varphi(O \eta)]) \\
& \leq \beta(\theta(h(\xi, \eta, \varrho, O, R, \varphi))) \theta(h(\xi, \eta, \varrho, O, R, \varphi)) \\
& \quad+L \theta(q(\xi, \eta, \varrho, O, R, \varphi))
\end{aligned}
$$

with $L \geq 0,1 / s+L<1$ and $s=4, p=2$.

In summary, all requirements of Theorem 14 are satisfied. $O$ and $R$ have a unique common fixed point $1 / 5$. result.

In Theorem 14, letting $\varphi=0$, we can obtain the following

Corollary 15. Let $(\mathscr{M}, \rho)$ be a complete rectangular $b$-metric space with coefficient $s \geq 1$. Let $\alpha: \mathscr{M} \times \mathscr{M} \longrightarrow[0,+\infty)$ and $O, R: \mathscr{M} \longrightarrow \mathscr{M}$ be given mappings with $O(\mathscr{M}) \subset R(\mathscr{M})$ and $R(\mathscr{M})$ is closed. Assume that $p \geq 2$ is a arbitrary constant and $\varphi: \mathscr{M} \longrightarrow[0,+\infty)$ is a lower semi-continuous function. If

(i) $O$ is $R-\alpha_{s^{p}}$-admissible

(ii) for $\xi, \eta \in M$ such that $\alpha(R \xi, R \eta) \geq s^{p}$ and $\mathrm{Q}(O \xi, O \eta)$ $\neq 0$, there exist $\theta \in \Theta, \beta \in \Omega$ and $L \geq 0,1 / s+L<1$ satisfying:

$\theta(\alpha(R \xi, R \eta) \mathrm{\varrho}(O \xi, O \eta)) \leq \beta(\theta(m(\xi, \eta, \varrho, O, R))) \theta(m(\xi, \eta, \varrho, O, R))$ $+\operatorname{L\theta }(n(\xi, \eta, \mathrm{\varrho}, O, R))$

where

$$
\begin{aligned}
m(\xi, \eta, \mathrm{\varrho}, O, R)= & \max \left\{\mathrm{\varrho}(O \xi, O \eta), \frac{\mathrm{\varrho}(O \eta, R \eta)}{1+\mathrm{\varrho}(O \xi, R \xi)}\right. \\
& \left.\cdot \mathrm{\varrho}(R \xi, R \eta), \frac{1}{2}(\mathrm{\varrho}(O \xi, R \eta)+\mathrm{\varrho}(R \xi, R \eta))\right\},
\end{aligned}
$$


and

$$
n(\xi, \eta, \varrho, O, R)=\frac{1}{2} \min \{\varrho(O \xi, O \eta), \varrho(R \xi, R \eta)\}
$$

(iii) there is $\xi_{0} \in \mathscr{M}$ such that $\alpha\left(R \xi_{0}, O \xi_{0}\right) \geq s^{p}$

(iv) properties $\left(A_{s^{p}}\right)$ and $\left(B_{s^{p}}\right)$ are fulfilled

(v) $\alpha$ satisfies transitive property, i.e., for $\xi, \eta, z \in \mathscr{M}$

$$
\alpha(\xi, \eta) \geq s^{p} \text { and } \alpha(\eta, z) \geq s^{p} \Rightarrow \alpha(\xi, z) \geq s^{p}
$$

then, $O$ and $R$ possess a unique point of coincidence in $\mathscr{M}$. Further, if $O$ and $R$ are weakly compatible, then $O$ and $R$ possess a unique common fixed point

If $\varphi=0, R=I$, and $L=0$ in Theorem 14, we have the following.

Corollary 16. Let $(\mathscr{M}, \mathrm{Q})$ be a complete rectangular $b$-metric space with coefficient $s \geq 2$ and $\mathscr{M}$ be closed. Let $\alpha: \mathscr{M} \times \mathscr{M}$ $\longrightarrow[0,+\infty)$ and $O: \mathscr{M} \longrightarrow \mathscr{M}$ be given mappings. Suppose $p \geq 2$ is a arbitrary constant. If

(i) $O$ is $\alpha_{s^{p}}$-admissible

(ii) for $\xi, \eta \in \mathscr{M}$ such that $\alpha(\xi, \eta) \geq s^{p}$ and $\mathrm{Q}(O \xi, O \eta) \neq 0$, there exists $\theta \in \Theta$ satisfying:

$$
\theta(\alpha(\xi, \eta) \mathrm{\varrho}(O \xi, O \eta)) \leq \beta \theta\left(m^{*}(\xi, \eta, \varrho, O)\right)
$$

where $\beta \in(0,1 / s)$ is a constant and

$$
m^{*}(\xi, \eta, \mathrm{\varrho}, O)=\max \left\{\mathrm{\varrho}(O \xi, O \eta), \frac{\mathrm{e}(O \eta, \eta)}{1+\mathrm{\varrho}(O \xi, \xi)} \cdot \mathrm{\varrho}(\xi, \eta), \frac{1}{2}(\mathrm{\varrho}(O \xi, \eta)+\mathrm{\varrho}(\xi, \eta))\right\}
$$

(iii) there is $\xi_{0} \in \mathscr{M}$ with $\alpha\left(\xi_{0}, O \xi_{0}\right) \geq s^{p}$

(iv) properties $\left(A_{s^{p}}\right)$ and $\left(B_{s^{p}}\right)$ are fulfilled when $R=I$

(v) $\alpha$ satisfies transitive property, i.e., for $\xi, \eta, z \in \mathscr{M}$

$$
\begin{gathered}
\alpha(\xi, \eta) \geq s^{p}, \\
\alpha(\eta, z) \geq s^{p} \Rightarrow \alpha(\xi, z) \geq s^{p},
\end{gathered}
$$

then $O$ possesses a unique fixed point

Definition 17 . The self-mappings $O, R: \mathscr{M} \longrightarrow \mathscr{M}$ are called $\alpha_{s}$ orbital admissible mapping, if the following condition holds:

$\alpha_{s}(\xi, O \xi) \geq s^{p}, \alpha_{s}(\xi, R \xi) \geq s^{p}$ imply $\alpha_{s}(O \xi, R O \xi) \geq s^{p}, \alpha_{s}(R \xi, O R \xi) \geq s^{p}$,

for a constant $p \geq 3$.

Definition 18. Let $O, R: \mathscr{M} \longrightarrow \mathscr{M}$ be two self-maps and $p$ $\geq 3$ be a real number. The pair $(O, R)$ is said to be triangular $\alpha_{s}$ orbital admissible if

(i) $O, R: \mathscr{M} \longrightarrow \mathscr{M}$ are $\alpha_{s}$ orbital admissible

(ii) $\alpha_{s}(\xi, \eta) \geq s^{p}, \alpha_{s}(\eta, O \eta) \geq s^{p}$ and $\alpha_{s}(\eta, R \eta) \geq s^{p} \quad$ imply $\alpha_{s}(\xi, O \eta) \geq s^{p}, \alpha_{s}(\xi, R \eta) \geq s^{p}$

Lemma 19. Let $O, R: \mathscr{M} \longrightarrow \mathscr{M}$ be two self-mappings satisfying the pair $(O, R)$ is triangular $\alpha_{s}$ orbital admissible. Suppose that there is $\xi_{0} \in \mathscr{M}$ satisfying $\alpha_{s}\left(\xi_{0}, O \xi_{0}\right) \geq s^{p}$. Define a sequence $\left\{\xi_{n}\right\}$ in $\mathscr{M}$ by $\xi_{2 i+1}=O \xi_{2 i}, \xi_{2 i+2}=R \xi_{2 i+1}$ where $i=$ $0,1,2, \cdots$. Then $\alpha_{s}\left(\xi_{n}, \xi_{m}\right) \geq s^{p}$ for $m, n \in \mathbb{N} \cup\{0\}$ with $m>$ $n$, .

Proof. Since $\alpha_{s}\left(\xi_{0}, O \xi_{0}\right)=\alpha_{s}\left(\xi_{0}, \xi_{1}\right) \geq s^{p}$ and $(O, R)$ is triangular $\alpha_{s}$ orbital admissible, $\alpha_{s}\left(\xi_{0}, O \xi_{0}\right) \geq s^{p}$ implies $\alpha_{s}\left(O \xi_{0}\right.$, $\left.R O \xi_{0}\right)=\alpha_{s}\left(\xi_{1}, R \xi_{1}\right)=\alpha_{s}\left(\xi_{1}, \xi_{2}\right) \geq s^{p}, \alpha_{s}\left(\xi_{1}, R \xi_{1}\right) \geq s^{p}$ implies $\alpha_{s}\left(R \xi_{1}, \mathrm{OR} \xi_{1}\right)=\alpha_{s}\left(\xi_{2}, O \xi_{2}\right)=\alpha_{s}\left(\xi_{2}, \xi_{3}\right) \geq s^{p}, \alpha_{s}\left(\xi_{2}, O \xi_{2}\right) \geq s^{p}$ implies $\alpha_{s}\left(O \xi_{2}, R O \xi_{2}\right)=\alpha_{s}\left(\xi_{3}, R \xi_{3}\right)=\alpha_{s}\left(\xi_{3}, \xi_{4}\right) \geq s^{p}$. Applying the above argument repeated, we obtain $\alpha_{s}\left(\xi_{n}, \xi_{n+1}\right) \geq$ $s^{p}$ for all $n \in \mathbb{N} \cup\{0\}$. Since $(O, R)$ is triangular $\alpha_{s}$ orbital admissible, $\alpha_{s}\left(\xi_{n}, \xi_{m}\right) \geq s^{p}$ for $n, m \in \mathbb{N} \cup\{0\}$ with $m>n$. $\square$

Definition 20. Let $(\mathscr{M}, \varrho)$ be a rectangular $b$-metric space with coefficient $s \geq 1$. Let $O, R: \mathscr{M} \longrightarrow \mathscr{M}$ be two selfmappings. Suppose that $\alpha_{s}: \mathscr{M} \times \mathscr{M} \longrightarrow[0,+\infty)$ and $\varphi$ $: \mathscr{M} \longrightarrow[0,+\infty)$ is a lower semicontinuous function and $p$ $\geq 3$ is an arbitrary constant. The mappings $O, R$ are said to be generalized $\alpha_{s}-\theta$-Geraghty contractions, if there exist $\theta \in \Theta, \beta, L \geq 0$ and $\beta+L<1,0<\lambda<1 / 4$ such that

$$
\begin{aligned}
& \alpha_{s}(\xi, \eta)[\mathrm{\varrho}(O \xi, R \eta)+\varphi(O \xi)+\varphi(R \eta)] \\
& \quad \leq \beta \theta(s(\xi, \eta, \varrho, O, R, \varphi))+L \theta(t(\xi, \eta, \varrho, O, \varphi)),
\end{aligned}
$$

for all $\xi, \eta \in \mathscr{M}$ with $\alpha_{s}(\xi, \eta) \geq s^{p}$ and $\mathrm{Q}(O \xi, R \eta)+\varphi(O \xi)+$ $\varphi(R \eta) \neq 0$, where

$$
\begin{aligned}
s(\xi, \eta, \mathrm{\varrho}, O, R, \varphi)= & \lambda \max \{\mathrm{\varrho}(\xi, \eta)+\varphi(\xi)+\varphi(\eta), \\
& \cdot \frac{1+\mathrm{\varrho}(\xi, O \xi)+\varphi(\xi)+\varphi(O \xi)}{1+\mathrm{\varrho}(\xi, \eta)+\varphi(\xi)+\varphi(\eta)} \\
& \cdot[\mathrm{\varrho}(O \xi, R \eta)+\varphi(O \xi)+\varphi(R \eta)], \\
& \cdot \frac{\mathrm{\varrho}(\eta, R \eta)+\varphi(\eta)+\varphi(R \eta)}{1+\mathrm{\varrho}(\xi, \eta)+\varphi(\xi)+\varphi(\eta)} \\
& \cdot[\mathrm{\varrho}(\xi, O \xi)+\varphi(\xi)+\varphi(O \xi)]\},
\end{aligned}
$$


$t(\xi, \eta, \varrho, O, \varphi)=\lambda \min \{\mathrm{\varrho}(\xi, O \xi)+\varphi(\xi)+\varphi(O \xi), \mathrm{\varrho}(\eta, O \xi)+\varphi(\eta)+\varphi(O \xi)\}$

Let $\alpha_{s}: \mathscr{M} \times \mathscr{M} \longrightarrow[0,+\infty)$ be a given mapping. Set.

$\left(C_{s^{p}}\right)$ For all $\xi^{*} \in \mathscr{M}$, we have $\alpha_{s}\left(\xi^{*}, \xi^{*}\right) \geq s^{p}$

$\left(D_{s^{p}}\right)$ For all $x, y \in C(O, R)$, one can get the condition of $\alpha_{s}(x, y) \geq s^{p}$ or $\alpha_{s}(y, x) \geq s^{p}$

Theorem 21. Let $(\mathscr{M}, \mathrm{Q})$ be a complete rectangular $b$-metric space with coefficient $s \geq 1$. Let $O, R: \mathscr{M} \longrightarrow \mathscr{M}$ be generalized $\alpha_{s}-\theta$-Geraghty contractions and one of $O$ and $R$ is continuous. If

(i) $O, R$ are triangular $\alpha_{s}$ orbital admissible

(ii) there is $\xi_{0} \in \mathscr{M}$ with satisfying $\alpha_{s}\left(\xi_{0}, O \xi_{0}\right) \geq s^{p}$

(iii) properties $\left(C_{s^{p}}\right)$ and $\left(D_{s^{p}}\right)$ are satisfied

then $O$ and $R$ possess a unique common fixed point

Proof. For $\xi_{0} \in \mathscr{M}$, define a sequence $\left\{\xi_{n}\right\}$ by $\xi_{2 j+1}=O \xi_{2 j}$, $\xi_{2 j+2}=R \xi_{2 j+1}$ for $j=0,1,2, \cdots$. We show that $O$ and $R$ have at most one common fixed point. Assume that $v \neq w$ are two common fixed points, then $R(v)=O(v)=v \neq w=R(w)$ $=O(w)$. Therefore, $\mathrm{Q}(O(v), R(w))=\mathrm{Q}(v, w)>0$. It follows from the property of $\left(D_{s^{p}}\right)$ that $\alpha_{s}(v, w) \geq s^{p}$ or $\alpha_{s}(w, v) \geq s^{p}$ . Without loss of generality, suppose that $\alpha_{s}(v, w) \geq s^{p}$. Letting $\xi=v$ and $\eta=w$ in (84), we have

$$
\begin{aligned}
\mathrm{\varrho}(v, w)+\varphi(v)+\varphi(w) & \leq s^{p}[\mathrm{\varrho}(O v, R w)+\varphi(O v)+\varphi(R w)] \\
& \leq \alpha_{s}(v, w)[\mathrm{\varrho}(O v, R w)+\varphi(O v)+\varphi(R w)] \\
& \leq \beta \theta(s(v, w, \varrho, O, R, \varphi))+L \theta(t(v, w, \varrho, O, \varphi))
\end{aligned}
$$

where

$$
\begin{aligned}
s(v, w, \mathrm{\varrho}, O, R, \varphi)= & \lambda \max \left\{\mathrm{\varrho}(v, w)+\varphi(v)+\varphi(w), \frac{1+\mathrm{\varrho}(v, O v)+\varphi(v)+\varphi(O v)}{1+\mathrm{\varrho}(v, w)+\varphi(v)+\varphi(w)}\right. \\
& \cdot[\mathrm{\varrho}(O v, R w)+\varphi(O v)+\varphi(R w)], \frac{\mathrm{\varrho}(w, R w)+\varphi(w)+\varphi(R w)}{1+\mathrm{\varrho}(v, w)+\varphi(v)+\varphi(w)} \\
& \cdot[\mathrm{\varrho}(v, O v)+\varphi(v)+\varphi(O v)]\} \\
< & \frac{1}{4} \max \{\mathrm{\varrho}(v, w)+\varphi(v)+\varphi(w), 2[\mathrm{\varrho}(v, w)+\varphi(v)+\varphi(w)], 4[\mathrm{\varrho}(v, w) \\
& +\varphi(v)+\varphi(w)]\}=\mathrm{\varrho}(v, w)+\varphi(v)+\varphi(w),
\end{aligned}
$$

and

$$
\begin{aligned}
t(v, w, \mathrm{\varrho}, O, \varphi) & =\lambda \min \{\mathrm{\varrho}(v, O v)+\varphi(v)+\varphi(O v), \mathrm{\varrho}(w, O v)+\varphi(w)+\varphi(O v)\} \\
& <\frac{1}{4} \min \{\mathrm{\varrho}(v, v)+\varphi(v)+\varphi(v), \mathrm{\varrho}(w, v)+\varphi(w)+\varphi(v)\} \\
& <\mathrm{\varrho}(v, w)+\varphi(v)+\varphi(w) .
\end{aligned}
$$

In view of (86), we have

$$
\begin{aligned}
\mathrm{\varrho}(v, w)+\varphi(v)+\varphi(w) & <\theta(\mathrm{\varrho}(v, w)+\varphi(v)+\varphi(w)) \\
& <\mathrm{\varrho}(v, w)+\varphi(v)+\varphi(w),
\end{aligned}
$$

which implies that $\mathrm{\varrho}(v, w)+\varphi(v)+\varphi(w)=0$. That is, $v=w$ and $\varphi(v)=0$. Hence, $O, R$ have at most one common fixed point.

Now, assume $\mathrm{Q}\left(\xi_{n}, \xi_{n+1}\right)>0$ for $n \in \mathbb{N}$. Otherwise, for some $k, \xi_{2 k}=\xi_{2 k+1}$, by assumption (ii) and Lemma 19, we have $\alpha_{s}\left(\xi_{2 k}, \xi_{2 k+1}\right) \geq s^{p}$. According to (84), if $\xi_{2 \mathrm{k}+1} \neq \xi_{2 k+2}$, we obtain

$$
\begin{aligned}
& \mathrm{\varrho}\left(\xi_{2 k+1}, \xi_{2 k+2}\right)+\varphi\left(\xi_{2 k+1}\right)+\varphi\left(\xi_{2 k+2}\right) \\
& \quad \leq s^{p}\left[\mathrm{\varrho}\left(O \xi_{2 k}, R \xi_{2 k+1}\right)+\varphi\left(O \xi_{2 k}\right)+\varphi\left(R \xi_{2 k+1}\right)\right] \\
& \quad \leq \alpha_{s}\left(\xi_{2 k}, \xi_{2 k+1}\right)\left[\mathrm{\varrho}\left(O \xi_{2 k}, R \xi_{2 k+1}\right)+\varphi\left(O \xi_{2 k}\right)+\varphi\left(R \xi_{2 k+1}\right)\right] \\
& \quad \leq \beta \theta\left(s\left(\xi_{2 k}, \xi_{2 k+1}, \varrho, O, R, \varphi\right)\right)+L \theta\left(t\left(\xi_{2 k}, \xi_{2 k+1}, \varrho, O, \varphi\right)\right),
\end{aligned}
$$

where

$$
\begin{aligned}
s\left(\xi_{2 k}, \xi_{2 k+1}, \varrho, O, R, \varphi\right)=\lambda & \max \left\{\mathrm{\varrho}\left(\xi_{2 k}, \xi_{2 k+1}\right)+\varphi\left(\xi_{2 k}\right)+\varphi\left(\xi_{2 k+1}\right),\right. \\
& \cdot \frac{1+\mathrm{\varrho}\left(\xi_{2 k}, O \xi_{2 k}\right)+\varphi\left(\xi_{2 k}\right)+\varphi\left(O \xi_{2 k}\right)}{1+\mathrm{\varrho}\left(\xi_{2 k}, \xi_{2 k+1}\right)+\varphi\left(\xi_{2 k}\right)+\varphi\left(\xi_{2 k+1}\right)} \\
& \cdot\left[\mathrm{\varrho}\left(O \xi_{2 k}, R \xi_{2 k+1}\right)+\varphi\left(O \xi_{2 k}\right)+\varphi\left(R \xi_{2 k+1}\right)\right], \\
& \cdot \frac{\mathrm{\varrho}\left(\xi_{2 k+1}, R \xi_{2 k+1}\right)+\varphi\left(\xi_{2 k+1}\right)+\varphi\left(R \xi_{2 k+1}\right)}{1+\mathrm{\varrho}\left(\xi_{2 k}, \xi_{2 k+1}\right)+\varphi\left(\xi_{2 k}\right)+\varphi\left(\xi_{2 k+1}\right)} \\
& \left.\cdot\left[\mathrm{\varrho}\left(\xi_{2 k}, O \xi_{2 k}\right)+\varphi\left(\xi_{2 k}\right)+\varphi\left(O \xi_{2 k}\right)\right]\right\} \\
< & \frac{1}{4} \max \left\{\mathrm{\varrho}\left(\xi_{2 k}, \xi_{2 k+1}\right)+\varphi\left(\xi_{2 k}\right)+\varphi\left(\xi_{2 k+1}\right),\right. \\
& \cdot \frac{1+\mathrm{\varrho}\left(\xi_{2 k}, \xi_{2 k+1}\right)+\varphi\left(\xi_{2 k}\right)+\varphi\left(\xi_{2 k+1}\right)}{1+\varrho\left(\xi_{2 k}, \xi_{2 k+1}\right)+\varphi\left(\xi_{2 k}\right)+\varphi\left(\xi_{2 k+1}\right)} \\
& \cdot\left[\mathrm{\varrho}\left(\xi_{2 k+1}, \xi_{2 k+2}\right)+\varphi\left(\xi_{2 k+1}\right)+\varphi\left(\xi_{2 k+2}\right)\right], \\
& \cdot \frac{\mathrm{\varrho}\left(\xi_{2 k+1}, \xi_{2 k+2}\right)+\varphi\left(\xi_{2 k+1}\right)+\varphi\left(\xi_{2 k+2}\right)}{1+\varrho\left(\xi_{2 k}, \xi_{2 k+1}\right)+\varphi\left(\xi_{2 k}\right)+\varphi\left(\xi_{2 k+1}\right)} \\
& \left.\cdot\left[\mathrm{\varrho}\left(\xi_{2 k}, \xi_{2 k+1}\right)+\varphi\left(\xi_{2 k}\right)+\varphi\left(\xi_{2 k+1}\right)\right]\right\} \\
\leq & \mathrm{\varrho}\left(\xi_{2 k+1}, \xi_{2 k+2}\right)+\varphi\left(\xi_{2 k+1}\right)+\varphi\left(\xi_{2 k+2}\right),
\end{aligned}
$$

$$
\begin{aligned}
t\left(\xi_{2 k}, \xi_{2 k+1}, \varrho, O, \varphi\right)= & \lambda \min \left\{\mathrm{\varrho}\left(\xi_{2 k}, O \xi_{2 k}\right)+\varphi\left(\xi_{2 k}\right)+\varphi\left(O \xi_{2 k}\right), \mathrm{\varrho}\left(\xi_{2 k+1}, O \xi_{2 k}\right)\right. \\
& \left.+\varphi\left(\xi_{2 k+1}\right)+\varphi\left(O \xi_{2 k}\right)\right\} \\
< & \frac{1}{4} \min \left\{\mathrm{\varrho}\left(\xi_{2 k}, \xi_{2 k+1}\right)+\varphi\left(\xi_{2 k}\right)+\varphi\left(\xi_{2 k+1}\right), \mathrm{\varrho}\left(\xi_{2 k+1}, \xi_{2 k+1}\right)\right. \\
& \left.+\varphi\left(\xi_{2 k+1}\right)+\varphi\left(\xi_{2 k+1}\right)\right\} \leq \mathrm{\varrho}\left(\xi_{2 k+1}, \xi_{2 k+2}\right)+\varphi\left(\xi_{2 k+1}\right)+\varphi\left(\xi_{2 k+2}\right) .
\end{aligned}
$$

In light of (90) and above inequalities, we have

$$
\begin{aligned}
& \mathrm{\varrho}\left(\xi_{2 k+1}, \xi_{2 k+2}\right)+\varphi\left(\xi_{2 k+1}\right)+\varphi\left(\xi_{2 k+2}\right) \leq \beta \theta\left(s\left(\xi_{2 k}, \xi_{2 k+1}, \mathrm{\varrho}, O, R, \varphi\right)\right) \\
& \quad+L \theta\left(t\left(\xi_{2 k}, \xi_{2 k+1}, \mathrm{\varrho}, O, \varphi\right)\right)+L \theta\left(\mathrm{\varrho}\left(\xi_{2 k+1}, \xi_{2 k+2}\right)+\varphi\left(\xi_{2 k+1}\right)+\varphi\left(\xi_{2 k+2}\right)\right) \\
& \quad<\theta\left(\mathrm{\varrho}\left(\xi_{2 k+1}, \xi_{2 k+2}\right)+\varphi\left(\xi_{2 k+1}\right)+\varphi\left(\xi_{2 k+2}\right)\right)<\mathrm{\varrho}\left(\xi_{2 k+1}, \xi_{2 k+2}\right)+\varphi\left(\xi_{2 k+1}\right) \\
& \quad+\varphi\left(\xi_{2 k+2}\right),
\end{aligned}
$$


which yields that $\mathrm{Q}\left(\xi_{2 k+1}, \xi_{2 k+2}\right)+\varphi\left(\xi_{2 k+1}\right)+\varphi\left(\xi_{2 k+2}\right)=0$. It follows that $\xi_{2 k+1}=\xi_{2 k+2}$.

Thus, $\xi_{2 k}$ is a common fixed point. Similarly, we can prove that $\xi_{2 k+1}$ is a common fixed point of $O$ and $R$ when $\xi_{2 k+1}=\xi_{2 k+2}$.

Now, assume that $\mathrm{\varrho}\left(\xi_{n}, \xi_{n+1}\right)>0$ for each $n \in \mathbb{N}$. Applying (84) with $\xi=\xi_{2 n}, \eta=\xi_{2 n+1}$, we get

$$
\begin{aligned}
& s\left(\varrho\left(\xi_{2 n+1}, \xi_{2 n+2}\right)+\varphi\left(\xi_{2 n+1}\right)+\varphi\left(\xi_{2 n+2}\right)\right) \\
& \quad \leq \beta \theta\left(s\left(\xi_{2 n}, \xi_{2 n+1}, \varrho, O, R, \varphi\right)\right)+L \theta\left(t\left(\xi_{2 n}, \xi_{2 n+1}, \varrho, O, \varphi\right)\right)
\end{aligned}
$$

where

$$
\begin{aligned}
s\left(\xi_{2 n}, \xi_{2 n+1}, \varrho, O, R, \varphi\right)= & \lambda \max \left\{\mathrm{\varrho}\left(\xi_{2 n}, \xi_{2 n+1}\right)+\varphi\left(\xi_{2 n}\right)+\varphi\left(\xi_{2 n+1}\right),\right. \\
& \cdot \frac{1+\varrho\left(\xi_{2 n}, \xi_{2 n+1}\right)+\varphi\left(\xi_{2 n}\right)+\varphi\left(\xi_{2 n+1}\right)}{1+\varrho\left(\xi_{2 n}, \xi_{2 n+1}\right)+\varphi\left(\xi_{2 n}\right)+\varphi\left(\xi_{2 n+1}\right)} \\
& \cdot\left[\mathrm{\varrho}\left(\xi_{2 n+1}, \xi_{2 n+2}\right)+\varphi\left(\xi_{2 n+1}\right)+\varphi\left(\xi_{2 n+2}\right)\right], \\
& \cdot \frac{\mathrm{\varrho}\left(\xi_{2 n+1}, \xi_{2 n+2}\right)+\varphi\left(\xi_{2 n+1}\right)+\varphi\left(\xi_{2 n+2}\right)}{1+\varrho\left(\xi_{2 n}, \xi_{2 n+1}\right)+\varphi\left(\xi_{2 n}\right)+\varphi\left(\xi_{2 n+1}\right)} \\
& \left.\cdot\left[\mathrm{\varrho}\left(\xi_{2 n}, \xi_{2 n+1}\right)+\varphi\left(\xi_{2 n}\right)+\varphi\left(\xi_{2 n+1}\right)\right]\right\} \\
< & \frac{1}{4} \max \left\{\mathrm{\varrho}\left(\xi_{2 n}, \xi_{2 n+1}\right)+\varphi\left(\xi_{2 n}\right)\right. \\
& \left.+\varphi\left(\xi_{2 n+1}\right), \varrho\left(\xi_{2 n+1}, \xi_{2 n+2}\right)+\varphi\left(\xi_{2 n+1}\right)+\varphi\left(\xi_{2 n+2}\right)\right\},
\end{aligned}
$$

$$
\begin{aligned}
t\left(\xi_{2 n}, \xi_{2 n+1}, \varrho, O, \varphi\right)= & \lambda \min \left\{\mathrm{\varrho}\left(\xi_{2 n}, \xi_{2 n+1}\right)+\varphi\left(\xi_{2 n}\right)\right. \\
& +\varphi\left(\xi_{2 n+1}\right), \varrho\left(\xi_{2 n+1}, \xi_{2 n+1}\right)+\varphi\left(\xi_{2 n+1}\right) \\
& \left.+\varphi\left(\xi_{2 n+1}\right)\right\}<\mathrm{\varrho}\left(\xi_{2 n}, \xi_{2 n+1}\right)+\varphi\left(\xi_{2 n}\right) \\
& +\varphi\left(\xi_{2 n+1}\right) .
\end{aligned}
$$

If $\mathrm{\varrho}\left(\xi_{2 n+1}, \xi_{2 n+2}\right)+\varphi\left(\xi_{2 n+1}\right)+\varphi\left(\xi_{2 n+2}\right)>\rho\left(\xi_{2 n}, \xi_{2 n+1}\right)+\varphi$ $\left(\xi_{2 n}\right)+\varphi\left(\xi_{2 n+1}\right)$ for some $n$, then by (94), (95), and (96), we have

$$
\begin{aligned}
& s\left(\mathrm{\varrho}\left(\xi_{2 n+1}, \xi_{2 n+2}\right)+\varphi\left(\xi_{2 n+1}\right)+\varphi\left(\xi_{2 n+2}\right)\right) \\
& \quad \leq \beta \theta\left(\mathrm{\varrho}\left(\xi_{2 n+1}, \xi_{2 n+2}\right)+\varphi\left(\xi_{2 n+1}\right)+\varphi\left(\xi_{2 n+2}\right)\right)+L \theta\left(\mathrm{\varrho}\left(\xi_{2 n+1}, \xi_{2 n+2}\right)\right. \\
& \left.\quad+\varphi\left(\xi_{2 n+1}\right)+\varphi\left(\xi_{2 n+2}\right)\right)<\theta\left(\mathrm{\varrho}\left(\xi_{2 n+1}, \xi_{2 n+2}\right)+\varphi\left(\xi_{2 n+1}\right)+\varphi\left(\xi_{2 n+2}\right)\right) \\
& \quad<\mathrm{Q}\left(\xi_{2 n+1}, \xi_{2 n+2}\right)+\varphi\left(\xi_{2 n+1}\right)+\varphi\left(\xi_{2 n+2}\right),
\end{aligned}
$$

which yields that

$$
\mathrm{Q}\left(\xi_{2 n+1}, \xi_{2 n+2}\right)+\varphi\left(\xi_{2 n+1}\right)+\varphi\left(\xi_{2 n+2}\right)=0 .
$$

That is, $\mathrm{\varrho}\left(\xi_{2 n+1}, \xi_{2 n+2}\right)=0$, a contradiction. Therefore, $\mathrm{\varrho}\left(\xi_{2 n+1}, \xi_{2 n+2}\right)+\varphi\left(\xi_{2 n+1}\right)+\varphi\left(\xi_{2 n+2}\right) \leq \varrho\left(\xi_{2 n}, \xi_{2 n+1}\right)+\varphi\left(\xi_{2 n}\right)+\varphi\left(\xi_{2 n+1}\right)$,

for $n \in \mathbb{N}$. It follows from (94),(95), and (96) that $s\left(\mathrm{\varrho}\left(\xi_{2 n+1}, \xi_{2 n+2}\right)+\varphi\left(\xi_{2 n+1}\right)+\varphi\left(\xi_{2 n+2}\right)\right)<\mathrm{\varrho}\left(\xi_{2 n}, \xi_{2 n+1}\right)+\varphi\left(\xi_{2 n}\right)+\varphi\left(\xi_{2 n+1}\right)$.
Using the same technique, we have

$s\left(\mathrm{\varrho}\left(\xi_{2 n+2}, \xi_{2 n+3}\right)+\varphi\left(\xi_{2 n+2}\right)+\varphi\left(\xi_{2 n+3}\right)\right)<\mathrm{\varrho}\left(\xi_{2 n+1}, \xi_{2 n+2}\right)+\varphi\left(\xi_{2 n+1}\right)+\varphi\left(\xi_{2 n+2}\right)$,

which implies that $\left\{\mathrm{Q}\left(\xi_{n}, \xi_{n+1}\right)+\varphi\left(\xi_{n}\right)+\varphi\left(\xi_{n+1}\right)\right\}$ is a nonincreasing sequence satisfying

$s\left(\mathrm{\varrho}\left(\xi_{n+1}, \xi_{n+2}\right)+\varphi\left(\xi_{n+1}\right)+\varphi\left(\xi_{n+2}\right)\right)<\mathrm{\varrho}\left(\xi_{n}, \xi_{n+1}\right)+\varphi\left(\xi_{n}\right)+\varphi\left(\xi_{n+1}\right)$.

So there exists a $\lambda \geq 0$ satisfying

$$
\lim _{n \longrightarrow+\infty}\left(\varrho\left(\xi_{n}, \xi_{n+1}\right)+\varphi\left(\xi_{n}\right)+\varphi\left(\xi_{n+1}\right)\right)=\lambda .
$$

Now, we suppose $\lambda>0$. By virtue of (94), (95), (96), and (99), one can get that

$$
\begin{gathered}
\mathrm{Q}\left(\xi_{2 n+1}, \xi_{2 n+2}\right)+\varphi\left(\xi_{2 n+1}\right)+\varphi\left(\xi_{2 n+2}\right) \leq \beta \theta\left(\mathrm{\varrho}\left(\xi_{2 n}, \xi_{2 n+1}\right)+\varphi\left(\xi_{2 n}\right)\right. \\
\left.+\varphi\left(\xi_{2 n+1}\right)\right)+L \theta\left(\mathrm{\varrho}\left(\xi_{2 n}, \xi_{2 n+1}\right)+\varphi\left(\xi_{2 n}\right)+\varphi\left(\xi_{2 n+1}\right) .\right.
\end{gathered}
$$

Letting $n \longrightarrow+\infty$ in (104), we have

$$
\begin{aligned}
\lambda= & \lim _{n \longrightarrow+\infty}\left(\mathrm{\varrho}\left(\xi_{2 n+1}, \xi_{2 n+2}\right)+\varphi\left(\xi_{2 n+1}\right)+\varphi\left(\xi_{2 n+2}\right)\right) \\
\leq & \beta \lim _{n \longrightarrow+\infty} \theta\left(\mathrm{\varrho}\left(\xi_{2 n}, \xi_{2 n+1}\right)+\varphi\left(\xi_{2 n}\right)+\varphi\left(\xi_{2 n+1}\right)\right) \\
& +L \lim _{n \longrightarrow+\infty} \theta\left(\mathrm{\varrho}\left(\xi_{2 n}, \xi_{2 n+1}\right)+\varphi\left(\xi_{2 n}\right)+\varphi\left(\xi_{2 n+1}\right)\right) \\
< & \lim _{n \longrightarrow+\infty} \theta\left(\mathrm{\varrho}\left(\xi_{2 n+1}, \xi_{2 n+2}\right)+\varphi\left(\xi_{2 n+1}\right)+\varphi\left(\xi_{2 n+2}\right)\right)=\theta(\lambda)<\lambda,
\end{aligned}
$$

a contradiction. It follows that

$$
\lim _{n \longrightarrow+\infty}\left(\varrho\left(\xi_{n}, \xi_{n+1}\right)+\varphi\left(\xi_{n}\right)+\varphi\left(\xi_{n+1}\right)\right)=0 .
$$

Hence,

$$
\lim _{n \longrightarrow+\infty} \mathrm{Q}\left(\xi_{n}, \xi_{n+1}\right)=0, \lim _{n \longrightarrow+\infty} \varphi\left(\xi_{n}\right)=0 .
$$

Now, we will show that $\left\{\xi_{n}\right\}$ is Cauchy. It is sufficient to show that $\left\{\xi_{3 n}\right\},\left\{\xi_{3 n+1}\right\}$, and $\left\{\xi_{3 n+2}\right\}$ are Cauchy. First of all, we prove $\left\{\xi_{3 n}\right\}$ is Cauchy. We take into consideration the following.: 
Case 1. $k=2 m+1$, where $m \geq 1$ and $3 n$ is an odd number. By means of rectangular inequality and (102), we deduce that

$$
\begin{aligned}
\mathrm{\varrho}\left(\xi_{3 n}, \xi_{3 n+3 k}\right) \leq & s\left[\mathrm{\varrho}\left(\xi_{3 n}, \xi_{3 n+1}\right)+\mathrm{\varrho}\left(\xi_{3 n+1}, \xi_{3 n+2}\right)+\mathrm{\varrho}\left(\xi_{3 n+2}, \xi_{3 n+3 k}\right)\right] \\
\leq & s\left[\mathrm{\varrho}\left(\xi_{3 n}, \xi_{3 n+1}\right)+\mathrm{\varrho}\left(\xi_{3 n+1}, \xi_{3 n+2}\right)\right]+s^{2}\left[\mathrm{\varrho}\left(\xi_{3 n+2}, \xi_{3 n+3}\right)\right. \\
& \left.+\mathrm{\varrho}\left(\xi_{3 n+3}, \xi_{3 n+4}\right)+\mathrm{\varrho}\left(\xi_{3 n+4}, \xi_{3 n+3 k}\right)\right] \leq s\left[\mathrm{\varrho}\left(\xi_{3 n}, \xi_{3 n+1}\right)\right. \\
& \left.+\mathrm{\varrho}\left(\xi_{3 n+1}, \xi_{3 n+2}\right)\right]+s^{2}\left[\mathrm{\varrho}\left(\xi_{3 n+2}, \xi_{3 n+3}\right)+\mathrm{\varrho}\left(\xi_{3 n+3}, \xi_{3 n+4}\right)\right] \\
& +s^{3}\left[\mathrm{\varrho}\left(\xi_{3 n+4}, \xi_{3 n+5}\right)+\mathrm{\varrho}\left(\xi_{3 n+5}, \xi_{3 n+6}\right)\right]+\cdots \cdots \\
& +s^{3 m+1}\left[\mathrm{\varrho}\left(\xi_{3 n+3(2 m+1)-3}, \xi_{3 n+3(2 m+1)-2}\right)\right. \\
& \left.+\mathrm{\varrho}\left(\xi_{3 n+3(2 m+1)-2}, \xi_{3 n+3(2 m+1)-1}\right)+\mathrm{\varrho}\left(\xi_{3 n+3(2 m+1)-1}, \xi_{3 n+3(2 m+1)}\right)\right] \\
\leq & s\left(\left(\frac{1}{s}\right)^{3 n}+\left(\frac{1}{s}\right)^{3 n+1}\right)+s^{2}\left(\left(\frac{1}{s}\right)^{3 n+2}+\left(\frac{1}{s}\right)^{3 n+3}\right) \\
& \left.+\cdots+s \cdot s^{3 m+1} \cdot\left(\frac{1}{s}\right)^{3 n+6 m+2}\right]\left(\mathrm{\varrho}\left(\xi_{0}, \xi_{1}\right)+\varphi\left(\xi_{0}\right)+\varphi\left(\xi_{1}\right)\right) \\
\leq & \left(\frac{1}{s}\right)^{3 n}\left[1+\frac{1}{s}+\left(\frac{1}{s}\right)^{2}+\cdots\right]\left(\mathrm{\varrho}\left(\xi_{0}, \xi_{1}\right)+\varphi\left(\xi_{0}\right)+\varphi\left(\xi_{1}\right)\right) \\
& +s\left(\frac{1}{s}\right)^{3 n+1}\left[1+\frac{1}{s}+\left(\frac{1}{s}\right)^{2}+\cdots\right]\left(\mathrm{\varrho}\left(\xi_{0}, \xi_{1}\right)+\varphi\left(\xi_{0}\right)+\varphi\left(\xi_{1}\right)\right) \\
= & \left(\frac{1}{s}\right)^{3 n} \cdot \frac{1+s}{1-1 / s}\left(\mathrm{\varrho}\left(\xi_{0}, \xi_{1}\right)+\varphi\left(\xi_{0}\right)+\varphi\left(\xi_{1}\right)\right) \longrightarrow 0 \text { as } \longrightarrow \infty
\end{aligned}
$$

The case that $3 n$ is an even number is similar to the case that $3 n$ is an odd number.

Case 2. $k=2 m$, where $m \geq 1$ and $3 n$ is an odd number. In view of rectangular inequality again, we get that

$$
\begin{aligned}
\mathrm{\varrho}\left(\xi_{3 n}, \xi_{3 n+3 k}\right) \leq & s\left[\mathrm{\varrho}\left(\xi_{3 n}, \xi_{3 n+1}\right)+\mathrm{\varrho}\left(\xi_{3 n+1}, \xi_{3 n+2}\right)+\mathrm{\varrho}\left(\xi_{3 n+2}, \xi_{3 n+3 k}\right)\right] \\
\leq & s\left[\mathrm{\varrho}\left(\xi_{3 n}, \xi_{3 n+1}\right)+\mathrm{\varrho}\left(\xi_{3 n+1}, \xi_{3 n+2}\right)\right]+s^{2}\left[\mathrm{\varrho}\left(\xi_{3 n+2}, \xi_{3 n+3}\right)\right. \\
& \left.+\mathrm{\varrho}\left(\xi_{3 n+3}, \xi_{3 n+4}\right)+\mathrm{\varrho}\left(\xi_{3 n+4}, \xi_{3 n+3 k}\right)\right] \\
\leq & s\left[\mathrm{\varrho}\left(\xi_{3 n}, \xi_{3 n+1}\right)+\mathrm{\varrho}\left(\xi_{3 n+1}, \xi_{3 n+2}\right)\right] \\
& +s^{2}\left[\mathrm{\varrho}\left(\xi_{3 n+2}, \xi_{3 n+3}\right)+\mathrm{\varrho}\left(\xi_{3 n+3}, \xi_{3 n+4}\right)\right] \\
& +s^{3}\left[\mathrm{\varrho}\left(\xi_{3 n+4}, \xi_{3 n+5}\right)+\mathrm{\varrho}\left(\xi_{3 n+5}, \xi_{3 n+6}\right)\right] \\
& +\cdots \cdots+s^{3 m}\left[\mathrm{\varrho}\left(\xi_{3 n+6 m-3}, \xi_{3 n+6 m-2}\right)+\mathrm{\varrho}\left(\xi_{3 n+6 m-2}, \xi_{3 n+6 m-1}\right)\right. \\
& \left.+\mathrm{\varrho}\left(\xi_{3 n+6 m-1}, \xi_{3 n+6 m)}\right)\right] \leq\left[\left(\left(\frac{1}{s}\right)^{3 n}+\left(\frac{1}{s}\right)^{3 n+1}\right)\right. \\
& \left.+s^{2}\left(\left(\frac{1}{s}\right)^{3 n+2}+\left(\frac{1}{s}\right)^{3 n+3}\right)+\cdots+s \cdot s^{3 m+1} \cdot\left(\frac{1}{s}\right)^{3 n+6 m+2}\right]\left(\mathrm{\varrho}\left(\xi_{0}, \xi_{1}\right)\right. \\
& \left.+\varphi\left(\xi_{0}\right)+\varphi\left(\xi_{1}\right)\right) \leq s\left(\frac{1}{s}\right)^{3 n}\left[1+\frac{1}{s}+\left(\frac{1}{s}\right)^{2}+\cdots\right]\left(\mathrm{\varrho}\left(\xi_{0}, \xi_{1}\right)+\varphi\left(\xi_{0}\right)\right. \\
& \left.+\varphi\left(\xi_{1}\right)\right)+s\left(\frac{1}{s}\right)^{3 n+1}\left[1+\frac{1}{s}+\left(\frac{1}{s}\right)^{2}+\cdots\right]\left(\mathrm{\varrho}\left(\xi_{0}, \xi_{1}\right)+\varphi\left(\xi_{0}\right)+\varphi\left(\xi_{1}\right)\right) \\
= & \left(\frac{1}{s}\right)^{3 n} \cdot \frac{1+s}{1-(1 / s)}\left(\mathrm{\varrho}\left(\xi_{0}, \xi_{1}\right)+\varphi\left(\xi_{0}\right)+\varphi\left(\xi_{1}\right)\right) \longrightarrow 0 \text { as } \longrightarrow \infty .
\end{aligned}
$$

The case that $3 n$ is an even number is similar to the case that $3 n$ is an odd number.

Hence, $\left\{\xi_{3 n}\right\}$ is a Cauchy sequence. Similarly, $\left\{\xi_{3 n+1}\right\}$, $\left\{\xi_{3 n+2}\right\}$ are also Cauchy sequences. That is, $\left\{\xi_{n}\right\}$ is Cauchy. According to the completeness of $(\mathscr{M}, \rho)$, we obtain that there is a $\xi^{*}$ in $\mathscr{M}$ satisfying

$$
\lim _{n \longrightarrow+\infty} O \xi_{2 n}=\lim _{n \longrightarrow+\infty} R \xi_{2 n+1}=\xi^{*}
$$

In view of the definition of $\varphi$, we have

$$
\varphi\left(\xi^{*}\right) \leq \liminf _{n \longrightarrow+\infty} \varphi\left(\xi_{n}\right)=0
$$

Next, we show that $O \xi^{*}=R \xi^{*}=\xi^{*}$ provided $O$ or $R$ is continuous. Without loss of generality, assume that $O$ is continuous. By (110), we get

$$
\xi^{*}=\lim _{n \longrightarrow+\infty} O \xi_{2 n}=O\left(\lim _{n \longrightarrow+\infty} \xi_{2 n}\right)=O\left(\xi^{*}\right) .
$$

This implies that $\xi^{*}$ is a fixed point of $O$.

Using property $\left(C_{s^{p}}\right)$, we have $\alpha_{s}\left(\xi^{*}, \xi^{*}\right) \geq s^{p}$. If $\xi^{*} \neq R \xi^{*}$, from condition (84), one can deduce

$$
\begin{aligned}
\mathrm{\varrho}\left(\xi^{*}, R \xi^{*}\right)+\varphi\left(\xi^{*}\right)+\varphi\left(R \xi^{*}\right) & \leq s\left[\varrho\left(O \xi^{*}, R \xi^{*}\right)+\varphi\left(O \xi^{*}\right)+\varphi\left(R \xi^{*}\right)\right] \\
& \leq \alpha_{s}\left(\xi^{*}, \xi^{*}\right)\left[\varrho\left(O \xi^{*}, R \xi^{*}\right)+\varphi\left(O \xi^{*}\right)+\varphi\left(R \xi^{*}\right)\right] \\
& \leq \beta \theta\left(s\left(\xi^{*}, \xi^{*}, \varrho, O, R, \varphi\right)\right)+L \theta\left(t\left(\xi^{*}, \xi^{*}, \varrho, O, \varphi\right)\right),
\end{aligned}
$$

where

$$
\begin{aligned}
& s\left(\xi^{*}, \xi^{*}, \mathrm{\varrho}, O, R, \varphi\right)= \lambda \max \left\{\mathrm{\varrho}\left(\xi^{*}, \xi^{*}\right)+\varphi\left(\xi^{*}\right)+\varphi\left(\xi^{*}\right),\right. \\
& \cdot \frac{1+\mathrm{\varrho}\left(\xi^{*}, O \xi^{*}\right)+\varphi\left(\xi^{*}\right)+\varphi\left(O \xi^{*}\right)}{1+\mathrm{\varrho}\left(\xi^{*}, \xi^{*}\right)+\varphi\left(\xi^{*}\right)+\varphi\left(\xi^{*}\right)} \\
& \cdot\left[\mathrm{\varrho}\left(O \xi^{*}, R \xi^{*}\right)+\varphi\left(O \xi^{*}\right)+\varphi\left(R \xi^{*}\right)\right], \\
& \cdot \frac{\mathrm{\varrho}\left(\xi^{*}, R \xi^{*}\right)+\varphi\left(\xi^{*}\right)+\varphi\left(R \xi^{*}\right)}{1+\mathrm{\varrho}\left(\xi^{*}, \xi^{*}\right)+\varphi\left(\xi^{*}\right)+\varphi\left(\xi^{*}\right)} \\
&\left.\cdot\left[\mathrm{\varrho}\left(\xi^{*}, O \xi^{*}\right)+\varphi\left(\xi^{*}\right)+\varphi\left(O \xi^{*}\right)\right]\right\} \\
&<\mathrm{\varrho}\left(\xi^{*}, R \xi^{*}\right)+\varphi\left(R \xi^{*}\right), \\
& t\left(\xi^{*}, \xi^{*}, \mathrm{\varrho}, O, \varphi\right)= 0 \leq \mathrm{\varrho}\left(\xi^{*}, R \xi^{*}\right)+\varphi\left(R \xi^{*}\right) .
\end{aligned}
$$

It follows from (113) that

$$
\begin{aligned}
\mathrm{\varrho}\left(\xi^{*}, R \xi^{*}\right)+\varphi\left(R \xi^{*}\right) \leq & \beta \theta\left(\rho\left(\xi^{*}, R \xi^{*}\right)+\varphi\left(R \xi^{*}\right)\right)+L \theta\left(\mathrm{\varrho}\left(\xi^{*}, R \xi^{*}\right)\right. \\
& \left.+\varphi\left(R \xi^{*}\right)\right)<\theta\left(\mathrm{\varrho}\left(\xi^{*}, R \xi^{*}\right)+\varphi\left(R \xi^{*}\right)\right) \\
< & \mathrm{\varrho}\left(\xi^{*}, R \xi^{*}\right)+\varphi\left(R \xi^{*}\right),
\end{aligned}
$$

which implies that $\mathrm{Q}\left(\xi^{*}, R \xi^{*}\right)+\varphi\left(R \xi^{*}\right)=0$, that is, $\xi^{*}=R \xi^{*}$ and $\varphi\left(R \xi^{*}\right)=0$. It follows that $O$ and $R$ possess the unique common fixed point $\xi^{*}$. This completes the proof.

Example 4. Let $\mathscr{M}=[0,+\infty)$ and $\mathrm{\varrho}(\xi, \eta)=(\xi-\eta)^{2}$. Define mappings $O, R: \mathscr{M} \longrightarrow \mathscr{M}$ by

$$
O \xi=\frac{\xi}{72}, R \xi=\frac{\xi}{63}, \xi \in[0,+\infty) \text {. }
$$

Define mappings $\alpha_{s}: \mathscr{M} \times \mathscr{M} \longrightarrow[0,+\infty)$ by

$$
\alpha_{s}(\xi, \eta)=s^{3}, \xi, \eta \in[0,+\infty) .
$$


Define $\theta:[0,+\infty) \longrightarrow[0,+\infty)$ and $\varphi: \mathscr{M} \longrightarrow \mathscr{M}$ with $\theta(\xi)=\xi / 2, \varphi(\xi)=\xi^{2}$. Let $\beta=1 / 3$ and $\lambda=1 / 6$.

For $\xi, \eta \in \mathscr{M}$ with $\alpha_{s}(\xi, \eta) \geq s^{3}$, we can know that for $\xi$, $\eta \in[0,+\infty)$ such that $\xi \neq 0$ or $\eta \neq 0$,

$$
\begin{aligned}
\alpha_{s}(\xi, \eta)[\mathrm{\varrho}(O \xi, R \eta)+\varphi(O \xi)+\varphi(R \eta)] & =3^{3} \cdot\left[\left(\frac{\xi}{72}-\frac{\eta}{63}\right)^{2}+\left(\frac{\xi}{72}\right)^{2}+\left(\frac{\eta}{63}\right)^{2}\right] \\
& =27 \cdot \frac{1}{81} \cdot \frac{3}{49}\left(\frac{49 \xi^{2}}{64}+\eta^{2}\right) \leq \frac{1}{49}\left(\xi^{2}+\eta^{2}\right),
\end{aligned}
$$

$\beta \theta(s(\xi, \eta, \varrho, O, R, \varphi))+L \theta(t(\xi, \eta, \varrho, O, \varphi)) \geq \beta \theta(s(\xi, \eta, \varrho, O, R, \varphi))$

$\geq \frac{1}{3} \cdot \frac{1}{2} \cdot \frac{1}{6}\left[(\varrho(\xi, \eta)+\varphi(\xi)+\varphi(\eta)]=\frac{1}{36}\left[(\xi-\eta)^{2}+\xi^{2}+\eta^{2}\right]\right.$

$\geq \frac{1}{36}\left(\xi^{2}+\eta^{2}\right)$.

In view of above inequalities, one can obtain that

$$
\begin{aligned}
& \alpha_{s}(\xi, \eta)[\varrho(O \xi, R \eta)+\varphi(O \xi)+\varphi(R \eta)] \leq \beta \theta(s(\xi, \eta, \varrho, O, R, \varphi)) \\
& \quad+L \theta(t(\xi, \eta, \varrho, O, \varphi)),
\end{aligned}
$$

for $\xi, \eta \in \mathscr{M}$ with $\alpha_{s}(\xi, \eta) \geq s^{p}$ and $\mathrm{\varrho}(O \xi, R \eta)+\varphi(O \xi)+\varphi(R$ $\eta) \neq 0$. Hence, all requirements of Theorem 21 are fulfilled with $p=3, s=3$, and $L<2 / 3$. It is obvious that $O$ and $R$ possess the unique common fixed point 0 .

Taking $\varphi=0$ in Theorem 21, one can obtain the following result.

Corollary 22. Let $(\mathscr{M}, \varrho)$ be a complete rectangular $b$-metric space with coefficient $s \geq 1$. Suppose $O, R: \mathscr{M} \longrightarrow \mathscr{M}$ are two given mappings and one of $O$ and $R$ is a continuous mapping. Assume that $\alpha_{s}: \mathscr{M} \times \mathscr{M} \longrightarrow[0,+\infty)$ and $p \geq 1$ is a constant and there exist $\theta \in \Theta, \beta, L \geq 0$ and $\beta+L<1,0<\lambda<1 / 4$ such that

$$
\alpha_{s}(\xi, \eta) \mathrm{\varrho}(O \xi, R \eta) \leq \beta \theta\left(s^{*}(\xi, \eta, \mathrm{\varrho}, O, R)\right)+L \theta\left(t^{*}(\xi, \eta, \varrho, O)\right),
$$

for $\xi, \eta \in \mathscr{M}$ with $\alpha_{s}(\xi, \eta) \geq s^{p}$ and $\mathrm{Q}(O \xi, R \eta) \neq 0$, where

$$
\begin{aligned}
& s^{*}(\xi, \eta, \mathrm{\varrho}, O, R)=\lambda \max \{\mathrm{\varrho}(\xi, \eta)\left.\frac{1+\mathrm{\varrho}(\xi, O \xi)}{1+\mathrm{\varrho}(\xi, \eta)} \cdot \mathrm{\varrho}(O \xi, R \eta), \frac{\mathrm{\varrho}(\eta, R \eta)}{1+\mathrm{\varrho}(\xi, \eta)} \cdot \mathrm{\varrho}(\xi, O \xi)\right\}, \\
& t^{*}(\xi, \eta, \mathrm{\varrho}, O)=\lambda \min \{\mathrm{\varrho}(\xi, O \xi), \mathrm{\varrho}(\eta, O \xi)\} .
\end{aligned}
$$

If

(i) $O, R$ are triangular $\alpha_{s}$ orbital admissible

(ii) there is $\xi_{0} \in \mathscr{M}$ with satisfying $\alpha_{s}\left(\xi_{0}, O \xi_{0}\right) \geq s^{p}$

(iii) properties $\left(C_{s^{p}}\right)$ and $\left(D_{s^{p}}\right)$ are satisfied then $O$ and $R$ possess a unique common fixed point

\section{Application}

In this part, we shall prove the existence and uniqueness of solution to the integral equation:

$$
\xi(\vartheta)=\int_{0}^{K} F(\vartheta, \lambda, \xi(\lambda)) d \lambda
$$

Let $\mathscr{M}=C([0, K])$ denote the collection of all continuous mappings on $[0, K]$. For $p \geq 2$, define

$$
\mathrm{Q}(\xi, \eta)=\sup _{\vartheta \in[0, K]}|\xi(\vartheta)-\eta(\vartheta)|^{p} \text { for all } \xi, \eta \in \mathscr{M}
$$

Hence, $(\mathscr{M}, \rho)$ is a complete rectangular $b$-metric space with $s=3^{p-1}$.

Theorem 23. Let $O: \mathscr{M} \longrightarrow \mathscr{M}$ by $O \xi(\vartheta)=\int_{0}^{K} F(\vartheta, \lambda, \xi(\lambda))$ $d \lambda$ and $\delta:(-\infty,+\infty) \times(-\infty,+\infty) \longrightarrow(-\infty,+\infty)$ be a given mapping. Suppose that

(i) $F:[0, K] \times[0, K] \times(-\infty,+\infty) \longrightarrow[0,+\infty) \quad$ is continuous

(ii) for all $\vartheta \in[0, K]$ and $\xi, \eta \in \mathscr{M}, \delta(\xi(\vartheta), \eta(\vartheta)) \geq 0$ implies $\delta(O \xi(\vartheta), O \eta(\vartheta)) \geq 0$

(iii) there exists $\xi_{0} \in \mathscr{M}$ satisfying $\delta\left(\xi_{0}(\vartheta), O \xi_{0}(\vartheta)\right) \geq 0$ for $\vartheta \in[0, K]$,

(iv) properties $\left(A_{s^{p}}\right)$ and $\left(B_{s^{p}}\right)$ are fulfilled when $R=I$

(v) there is a continuous mapping $\gamma:[0, K] \times[0, K]$ $\longrightarrow[0,+\infty)$ such that

$$
\sup _{\vartheta \in[0, K]} \int_{0}^{K} \gamma(\vartheta, \lambda) d \lambda \leq 1
$$

(vi) there exists a real number $\beta \in(0,1 / s)$ satisfying for $(\vartheta, \lambda) \in[0, K] \times[0, K]$

$$
|F(\vartheta, \lambda, \xi(\lambda))-F(\vartheta, \lambda, \eta(\lambda))| \leq \sqrt[p]{\frac{\beta}{2 s^{p}}} \gamma(\vartheta, \lambda)|\xi(\lambda)-\eta(\lambda)|
$$

Then, the integral equation (123) possesses a unique solution $\xi(\vartheta) \in \mathscr{M}$.

Proof. Define $\alpha: \mathscr{M} \times \mathscr{M} \longrightarrow[0,+\infty)$ by

$$
\alpha(\xi, \eta)= \begin{cases}s^{p}, & \text { if } \delta(\xi(\vartheta), \eta(\vartheta)) \geq 0 \\ 0, & \text { otherwise }\end{cases}
$$

For $\xi, \eta \in \mathscr{M}$, according to assumptions (i)-(vi), we 
obtain

$$
\begin{aligned}
s^{p} \mathrm{Q}(O \xi(\vartheta), O \eta(\vartheta))= & s^{p} \sup _{\vartheta \in[0, K]}|O \xi(\vartheta)-O \eta(\vartheta)|^{p} \\
= & s^{p} \sup _{\vartheta \in[0, K]} \mid \int_{0}^{K} F(\vartheta, \lambda, \xi(\lambda)) d \lambda \\
& -\left.\int_{0}^{K} F(\vartheta, \lambda, \eta(\lambda)) d \lambda\right|^{p} \\
\leq & s^{p} \sup _{\vartheta \in[0, K]}\left(\int_{0}^{K}|F(\vartheta, \lambda, \xi(\lambda))-F(\vartheta, \lambda, \eta(\lambda))| d \lambda\right)^{p} \\
\leq & s^{p} \sup _{\vartheta \in[0, K]}\left(\int_{0}^{K} \sqrt[p]{\frac{\beta}{2 s^{p}}} \gamma(\vartheta, \lambda)|\xi(\lambda)-\eta(\lambda)| d \lambda\right)^{p} \\
\leq & s^{p} \sup _{\vartheta \in[0, K]}\left(\int_{0}^{K} \sqrt[p]{\frac{\beta}{2 s^{p}}} \gamma(\vartheta, \lambda) d \lambda\right)^{p} \\
& \cdot \sup _{\vartheta \in[0, K]}|\xi(\vartheta)-\eta(\vartheta)|^{p} \\
= & \beta \cdot \frac{1}{2} \varrho(\xi, \eta) \leq \beta \theta\left(m^{*}(\xi, \eta, \varrho, O)\right) .
\end{aligned}
$$

Letting $\theta(\vartheta)=9 / 2$, one can verify that all the conditions of Corollary 16 hold. As a result, $O$ possesses a unique fixed point $\xi \in \mathscr{M}$, that is, $\xi(\vartheta)$ is the unique solution of integral equation (123). This completes the proof.

\section{Data Availability}

No data were used to support this study.

\section{Conflicts of Interest}

The authors declare that they have no conflicts of interest.

\section{Authors' Contributions}

All authors equally contributed to this paper and approved the final version.

\section{Acknowledgments}

The authors acknowledge the support by the Science and Research Project Foundation of Department of Education of Liaoning Province through Grant Nos. LQN201902 and LJC202003.

\section{References}

[1] S. Banach, "Sur les opérations dans les ensembles abstraits et leur application aux équations intégrales," Fundamenta Mathematicae, vol. 3, no. 3, pp. 133-181, 1922.

[2] S. Czerwik, "Contraction mappings in $b$ - metric spaces," Acta Mathematica et Informatica Universitatis Ostraviensis, vol. 1, pp. 5-11, 1993.

[3] V. Berinde, "Generalized contractions in quasimetric spaces," Seminar on Fixed Point Theory Preprint, vol. 3, pp. 3-9, 1993.

[4] M. B. Zada, M. Sarwar, and P. Kumam, "Fixed point results for rational type contraction in $b$-metric spaces," International
Journal of Analysis and Applications, vol. 16, no. 6, pp. $904-$ 920, 2018.

[5] M. Pacurar, "A fixed point result for $\phi$ - contractions and fixed points on $b$-metric spaces without the boundness assumption," Polytechnica Posnaniensis, vol. 43, pp. 127-136, 2010.

[6] H. Aydi, M. Bota, and S. Moradi, "A common fixed points for weak $\phi$-contractions on $b$ - metric spaces," Fixed Point Theory, vol. 13, pp. 337-346, 2012.

[7] S. Hussain, M. Sarwar, and C. Tunc, "Periodic fixed point theorems via rational type contraction in $b$ - metric spaces," Journal of Mathematical Analysis and Applications, vol. 10, no. 3, pp. 61-67, 2019.

[8] D. Gopal, P. Agarwal, and P. Kumam, Metric Structures and Fixed Point Theory, Chapman and Hall/CRC, New York, USA, 1st edition, 2021.

[9] M. Younis, D. Singh, and A. A. N. Abdou, "A fixed point approach for tuning circuit problem in dislocated $b$-metric spaces," Mathematical Methods in the Applied Sciences, 2021.

[10] M. Younis and D. Singh, "On the existence of the solution of Hammerstein integral equations and fractional differential equations," Journal of Applied Mathematics and Computing, 2021.

[11] M. Younis, D. Singh, I. Altun, and V. Chauhan, "Graphical structure of extended $b$-metric spaces: an application to the transverse oscillations of a homogeneous bar," International Journal of Nonlinear Sciences and Numerical Simulation, 2021.

[12] A. Branciari, "A fixed point theorem of Banach-Caccioppoli type on a class of generalized metric spaces," Publicationes Mathematiques, vol. 57, pp. 31-37, 2000.

[13] H. Lakzian and B. Samet, "Fixed points for $(\psi, \varphi)$ - weakly contractive mapping in generalised metric spaces," Applied Mathematics Letters, vol. 25, no. 5, pp. 902-906, 2012.

[14] I. M. Erhan, E. Karapinar, and T. Sekulic, "Fixed points of $(\psi$ $, \varphi)$ contractions on rectangular metric spaces," Fixed Point Theory and Applications, vol. 2012, Article ID 138, 2012.

[15] C. D. Bari and P. Vetro, "Common fixed points in generalized metric spaces," Applied Mathematics and Computation, vol. 218, no. 13, pp. 7322-7325, 2012.

[16] R. George and R. Rajagopalan, "Common fixed point results for $\psi-\varphi$ contractions in rectangular metric spaces," Bulletin of Mathematical Analysis and Applications, vol. 5, no. 1, pp. 44-52, 2013.

[17] J. Wang and J. I. Pei-Sheng, "Some fixed point theorems for new $\theta$ - contractions in complete rectangular-metric spaces," Journal of Qingdao University (Natural Science Edition), vol. 31, no. 4, pp. 10-15, 2018.

[18] L. B. Budhia, H. Aydi, A. H. Ansari, and D. Gopal, "Some new fixed point results in rectangular metric spaces with an application to fractional-order functional differential equations," Nonlinear Analysis: Modelling and Control, vol. 25, no. 4, pp. 580-597, 2020.

[19] M. Younis, D. Singh, and A. Goyal, “A novel approach of graphical rectangular $b$ - metric spaces with an application to the vibrations of a vertical heavy hanging cable," Journal of Fixed Point Theory and Applications, vol. 21, p. 33, 2019.

[20] M. Younis, D. Singh, and L. Shi, "Revisiting graphical rectangular b-metric spaces," Asian-European Journal of Mathematics, no. article 2250072, 2021.

[21] R. George, S. Radenovic, K. P. Reshma, and S. Shukla, "Rectangular b-metric space and contraction principles," Journal of 
Nonlinear Sciences and Applications, vol. 8, no. 6, pp. 10051013, 2015.

[22] Z. Kadelburg and S. Radenovic, "Pata-type common fixed point results in $b$-metric and $b$-rectangular metric spaces," Journal of Nonlinear Sciences and Applications, vol. 8, no. 6, pp. 944-954, 2015.

[23] Z. D. Mitrovic, "A note on a Banach's fixed point theorem in $b$ -rectangular metric space and $b$-metric space," Mathematica Slovaca, vol. 68, no. 5, pp. 1113-1116, 2018.

[24] H. S. Ding, M. Imdadb, S. Radenovic, and J. Vujakovic, "On some fixed point results in $b$ - metric, rectangular and $b-$ rectangular metric spaces," Arab Journal of Mathematical Sciences, vol. 22, no. 2, pp. 151-164, 2016.

[25] P. Sukprasert, P. Kumam, D. Thongtha, and K. Sombut, "Fixed point results on generalized $(\psi, \phi)_{s}$ - contractive mappings in rectangular $b$-metric spaces," Communications in Mathematics and Applications, vol. 7, no. 3, pp. 207-216, 2016.

[26] J. R. Roshan, V. Parvaneh, Z. Kadelburg, and N. Hussain, "New fixed point results in $b$-rectangular metric spaces," Nonlinear Analysis: Modelling and Control, vol. 21, no. 5, pp. 614634, 2016.

[27] Z. D. Mitrovic, "On an open problem in rectangular $b$-metric space," Journal of Analysis, vol. 25, no. 1, pp. 135-137, 2017.

[28] A. Sunarsini and S. D. Biahdillah, "Application of Banach contraction principle in complex valued rectangular $b$ - metric space," Journal of Physics Conference Series, vol. 1490, article 012003, 2020.

[29] R. George and K. P. Reshma, "Common couple fixed points of some generalised $T$-contractions in rectangular $b$-metric space and application," Advances in Fixed Point Theory, vol. 10, p. 20, 2020.

[30] M. Asim, M. Imdad, and S. Shukla, "Fixed point results for Geraghty-weak contractions in ordered partial rectangular $b$ -metric spaces," Afrika Matematika, vol. 32, no. 5-6, pp. 811-827, 2021.

[31] Y. Alber and S. Guerre-Delabriere, "Principles of weakly contractive maps in Hilbert spaces," in New Results in Operator Theory and Its Applications, I. Gohberg and Y. Lyubich, Eds., vol. 98 of Operator Theory: Advances and Applications, Birkhauser, Basel, 1997.

[32] B. Rhoades, "Some theorems on weakly contractive maps," Nonlinear Analysis, vol. 47, no. 4, pp. 2683-2693, 2001.

[33] P. Agarwa, M. Jleli, and B. Samet, Fixed Point Theory in Metric Spaces, Springer, Singapore, 1st edition, 2018.

[34] N. Jamal, T. Abdeljawad, M. Sarwar, N. Mlaiki, and P. Sumati Kumari, "Some valid generalizations of Boyd and Wong inequality and $\psi, \phi$-weak contraction in partially ordered $b$ -metric spaces," International Journal of Mathematics and Mathematical Sciences, vol. 2020, Article ID 9307302, 13 pages, 2020.

[35] Y. Hao and H. Guan, "On some common fixed point results for weakly contraction mappings with application," Journal of Function Spaces, vol. 2021, Article ID 5573983, 14 pages, 2021.

[36] G. Jungck, "Compatible mappings and common fixed points," International Journal of Mathematics and Mathematical Sciences, vol. 9, no. 4, p. 779, 1986.

[37] J. Matkkowski, "Fixed point theorems for mappings with a contractive iterate at a point," Proceedings of the American Mathematical Society, vol. 62, no. 2, pp. 344-348, 1977. 